\title{
A FINITE ELEMENT METHOD WITH LAGRANGE MULTIPLIERS FOR LOW-FREQUENCY HARMONIC MAXWELL EQUATIONS*
}

\author{
ALFREDO BERMÚDEZ ${ }^{\dagger}$, RODOLFO RODRÍGUEZ ${ }^{\ddagger}$ AND PILAR SALGADO§
}

\begin{abstract}
The aim of this paper is to analyze a finite element method to solve the low-frequency harmonic Maxwell equations in a bounded domain containing conductors and dielectrics. This system of partial differential equations is a model for the so-called eddy currents problem. After writing this problem in terms of the magnetic field, it is discretized by Nédélec edge finite elements on a tetrahedral mesh. Error estimates are easily obtained if the curl-free condition is imposed on the elements in the dielectric domain.

Then, the curl-free condition is imposed, at a discrete level, by introducing a piecewise linear multivalued potential. The resulting problem is shown to be a discrete version of other continuous formulation in which the magnetic field in the dielectric part of the domain has been replaced by a magnetic potential. Moreover, this approach leads to an important saving in computational effort. Problems related to the topology are also considered in that the possibility of having a non simply connected dielectric domain is taken into account.

Implementation issues are discussed, including an amenable procedure to impose the boundary conditions by means of a Lagrange multiplier. Finally the method is applied to solve a threedimensional model problem: a cylindrical electrode surrounded by dielectric.
\end{abstract}

Key words. low-frequency harmonic Maxwell equations, eddy currents problems, finite element computational electromagnetism

AMS subject classifications. $78 \mathrm{M} 10,65 \mathrm{~N} 30$

1. Introduction. In this paper we analyze a finite element method with Lagrange multipliers to solve the eddy currents model in a bounded domain including conductors and dielectrics. This model can be obtained from Maxwell equations by assuming that all fields are harmonic and the current frequency is low enough so that the term involving the displacement current in Ampère's Law can be neglected. Such a situation happens, for instance, in problems related with machines working at power frequencies. In particular, this paper is motivated by the need of a three-dimensional numerical simulation of a metallurgical furnace (see Bermúdez et al $[6,7]$ for related works concerning axisymmetric models).

Because of many interesting applications in electrical engineering, numerical solution of eddy currents problems became an important research area, leading to a great number of publications in recent years (see, for instance, $[2,3,8,9,10,12,13,16,20$, 30]. The books by Bossavit [11] and Silvester and Ferrari [29] also contain valuable material on this subject and include large reference lists.

While several papers deal with the mathematical and numerical analysis of the full harmonic Maxwell equations (see for instance the papers by Monk [23, 24, 25], and Fernandes and Gilardi [17]), the number of papers concerning analysis of the eddy

*This work was partially supported by Programa de Cooperación Científica con Iberoamérica, Ministerio de Educación y Ciencia, Spain.

${ }^{\dagger}$ Departamento de Matemática Aplicada, Universidade de Santiago de Compostela, 15706, Santiago de Compostela, Spain (mabermud@usc.es). Partially supported by Xunta de Galicia research project PGIDT00PXI20701PR (Spain).

${ }^{\ddagger} \mathrm{GI}^{2} \mathrm{MA}$, Departamento de Ingeniería Matemática, Universidad de Concepción, Casilla 160-C, Concepción, Chile (rodolfo@ing-mat.udec.cl). Partially supported by FONDAP in Applied Mathematics (Chile).

$\S$ Departamento de Matemática Aplicada, Universidade de Santiago de Compostela, 15706, Santiago de Compostela, Spain (mpilar@usc.es). Partially supported by Xunta de Galicia research project PGIDT00PXI20701PR (Spain). 
current model is much smaller. Significant mathematical and numerical results have been obtained by MacCamy and coauthors [18, 21, 22] for a two-dimensional eddy current problem. In the three-dimensional case, let us mention the article by Ammari et al [4], where a thorough justification of the eddy current model is given.

The above mentioned papers deal with the eddy currents problem in the whole space, the infinity being usually taken into account by means of integral equations. A useful alternative approach is considered by Alonso and Valli $[2,3]$, where the problem in a bounded domain is considered, including appropriate boundary conditions. In these papers, a formulation involving only the electric field is given and then numerically solved by using a domain decomposition technique and Nédélec edge finite elements.

In the present paper we also consider the eddy currents problem in a bounded domain which includes conductors and dielectrics. The conductors are not assumed to be totally included in the problem domain. We consider a formulation in terms of magnetic field with mixed Neumann and Dirichlet boundary conditions. The former are the natural conditions for the conducting part of the boundary. The latter are imposed on the dielectric part and allow taking into account all the electromagnetics effects outside the domain.

Then, following Bossavit and Verité [13], we introduce a scalar magnetic potential in the domain occupied by the dielectric. This hybrid formulation is discretized by using Nédélec edge elements for the magnetic field and standard piecewise linear continuous elements for the magnetic potential.

The outline of the paper is as follows: In Section 2, we recall the eddy currents model and obtain a weak formulation involving the magnetic field only. Section 3 concerns existence and uniqueness of solution which are proved by using classical tools. Then, in Section 4, we introduce a scalar magnetic potential in the dielectric domain and show that the resulting problem is completely equivalent to the previous one. The numerical discretization is introduced in Section 5, where error estimates are obtained under mild regularity assumption on the solution.

In order to solve the discretized problem, a Lagrange multiplier is proposed in Section 6 to impose the Dirichlet boundary conditions. The resulting mixed problem is shown to attain a unique solution and to be equivalent to the original discrete one. Finally, in Section 7, we report numerical results for a test with known analytical solution; these results confirm the predicted order of convergence of the method.

2. The eddy currents problem. Eddy currents are usually modeled by the low-frequency harmonic Maxwell equations. Let us recall first the governing equations of electromagnetism; namely, Maxwell equations:

$$
\begin{aligned}
\frac{\partial \mathcal{D}}{\partial t}-\operatorname{curl} \mathcal{H} & =-\mathcal{J}, \\
\frac{\partial \mathcal{B}}{\partial t}+\operatorname{curl} \mathcal{E} & =\mathbf{0}, \\
\operatorname{div} \mathcal{B} & =0, \\
\operatorname{div} \mathcal{D} & =\rho,
\end{aligned}
$$

constitutive laws:

$$
\begin{aligned}
\mathcal{B} & =\mu \mathcal{H}, \\
\mathcal{D} & =\epsilon \mathcal{E},
\end{aligned}
$$


and Ohm's law in conductors:

$$
\mathcal{J}=\sigma \mathcal{E}
$$

We have used notations which are standard in electromagnetism:

- $\mathcal{D}$ is the electric displacement,

- $\mathcal{E}$ is the electric field,

- $\mathcal{B}$ is the magnetic induction,

- $\mathcal{H}$ is the magnetic field,

- $\mathcal{J}$ is the current density,

- $\rho$ is the electric charge density,

- $\mu$ is the magnetic permeability,

- $\epsilon$ is the electric permittivity,

- $\sigma$ is the electric conductivity.

We use boldface letters to denote vector fields and variables, as well as vector-valued operators, throughout the paper.

When alternating currents are considered, all the fields have the following steadystate form:

$$
\mathcal{F}(\mathbf{x}, t)=\operatorname{Re}\left[\mathrm{e}^{\mathrm{i} \omega t} \mathbf{F}(\mathbf{x})\right]
$$

where $\omega$ is the angular frequency. Moreover, in the low-frequency harmonic regime, the term in (2.1) including the electric displacement can be neglected. Under these assumptions, equations (2.1)-(2.7) reduce to the so-called eddy currents model:

$$
\begin{aligned}
\operatorname{curl} \mathbf{H} & =\mathbf{J}, \\
\mathrm{i} \omega \mu \mathbf{H}+\operatorname{curl} \mathbf{E} & =\mathbf{0}, \\
\operatorname{div} \mathbf{B} & =0 \\
\operatorname{div} \mathbf{D} & =\rho,
\end{aligned}
$$

with

$$
\begin{aligned}
\mathbf{B} & =\mu \mathbf{H}, \\
\mathbf{D} & =\epsilon \mathbf{E}, \\
\mathbf{J} & =\sigma \mathbf{E} .
\end{aligned}
$$

We are interested in solving these equations in a bounded domain $\Omega$ which consists of two parts, $\Omega_{\mathrm{C}}$ and $\Omega_{\mathrm{D}}$, occupied by conductors and dielectrics, respectively. The electric conductivity $\sigma$ vanishes in the dielectric domain. The boundary of the domain $\Omega$ also splits into two parts: $\Gamma_{\mathrm{C}}:=\partial \Omega_{\mathrm{C}} \cap \partial \Omega$ and $\Gamma_{\mathrm{D}}:=\partial \Omega_{\mathrm{D}} \cap \partial \Omega$. Finally, we denote $\Gamma_{\mathrm{I}}:=\partial \Omega_{\mathrm{C}} \cap \partial \Omega_{\mathrm{D}}$, the interface between dielectric and conductors.

Boundary conditions must be added to solve the eddy currents model in the bounded domain $\Omega$. We consider:

$$
\begin{array}{ll}
\mathbf{E} \times \mathbf{n}=\mathbf{0} & \text { on } \Gamma_{\mathrm{C}}, \\
\mathbf{H} \times \mathbf{n}=\mathbf{f} & \text { on } \Gamma_{\mathrm{D}},
\end{array}
$$

with $\mathbf{f}$ being a given tangential vector field (i.e., satisfying $\mathbf{f} \cdot \mathbf{n}=0$ on $\Gamma_{\mathrm{D}}$ ).

In the equations above, $\mathbf{n}$ denotes the outer unit normal vector to $\partial \Omega$. Throughout the paper, $\mathbf{n}$ will denote a unit vector normal to a given surface, not necessarily the 
same at each occurrence. In general, it will not be explicitly mentioned which this surface is, provided this is sufficiently clear from the context.

To obtain a weak formulation of the boundary value problem (2.8)-(2.16), consider a test function $\mathbf{G}$ such that $\mathbf{G} \times \mathbf{n}=\mathbf{0}$ on $\Gamma_{\mathrm{D}}$. From (2.9) we have

$$
\mathrm{i} \omega \int_{\Omega} \mu \mathbf{H} \cdot \overline{\mathbf{G}}+\int_{\Omega} \operatorname{curl} \mathbf{E} \cdot \overline{\mathbf{G}}=0 .
$$

Now, we can transform the second term above by using a Green's formula:

$$
\begin{aligned}
\int_{\Omega} \operatorname{curl} \mathbf{E} \cdot \overline{\mathbf{G}} & =\int_{\Omega} \mathbf{E} \cdot \operatorname{curl} \overline{\mathbf{G}}-\int_{\Gamma_{\mathrm{C}}} \mathbf{E} \cdot \mathbf{n} \times \overline{\mathbf{G}} d \Gamma \\
& =\int_{\Omega} \mathbf{E} \cdot \operatorname{curl} \overline{\mathbf{G}}-\int_{\Gamma_{\mathrm{C}}} \mathbf{E} \times \mathbf{n} \cdot \overline{\mathbf{G}} d \Gamma=\int_{\Omega} \mathbf{E} \cdot \operatorname{curl} \overline{\mathbf{G}}
\end{aligned}
$$

where we have used the boundary condition (2.15) to obtain the last equality. We observe that equations (2.8) and (2.14), and the fact that $\sigma$ is null in the dielectric domain, lead to

$$
\operatorname{curl} \mathbf{H}=\mathbf{0} \quad \text { in } \Omega_{\mathrm{D}} .
$$

Because of this, we only need to take test functions $\mathbf{G}$ satisfying $\operatorname{curl} \mathbf{G}=\mathbf{0}$ in $\Omega_{\mathrm{D}}$. By doing so, equations (2.17) and (2.18) yield

$$
\mathrm{i} \omega \int_{\Omega} \mu \mathbf{H} \cdot \overline{\mathbf{G}}+\int_{\Omega_{\mathrm{C}}} \mathbf{E} \cdot \operatorname{curl} \overline{\mathbf{G}}=0
$$

Instead, in the conductors, equations (2.8) and (2.14) lead to $\mathbf{E}=\frac{1}{\sigma} \operatorname{curl} \mathbf{H}$, which allows us to eliminate $\mathbf{E}$ in the equation above. Thus, we finally obtain

$$
\mathrm{i} \omega \int_{\Omega} \mu \mathbf{H} \cdot \overline{\mathbf{G}}+\int_{\Omega_{\mathrm{C}}} \frac{1}{\sigma} \operatorname{curl} \mathbf{H} \cdot \operatorname{curl} \overline{\mathbf{G}}=0 .
$$

3. Analysis of the magnetic field formulation of the eddy currents problem. Let us assume that $\Omega$ is simply connected, with a Lipschitz-continuous connected boundary. The subdomains $\Omega_{\mathrm{C}}$ and $\Omega_{\mathrm{D}}$ are also assumed to have Lipschitzcontinuous boundaries, although not necessarily connected. Finally, the boundaries of $\Gamma_{\mathrm{C}}, \Gamma_{\mathrm{D}}$, and $\Gamma_{\mathrm{I}}$ are assumed to be Lipschitz-continuous too.

We use standard notation for Sobolev spaces and norms. Moreover, we recall the definition of some functional spaces. Let

$$
\mathrm{H}(\operatorname{curl}, \Omega):=\left\{\mathbf{G} \in \mathrm{L}^{2}(\Omega)^{3}: \operatorname{curl} \mathbf{G} \in \mathrm{L}^{2}(\Omega)^{3}\right\},
$$

endowed with the norm

$$
\|\mathbf{G}\|_{\mathrm{H}(\operatorname{curl}, \Omega)}:=\left[\|\mathbf{G}\|_{\mathrm{L}^{2}(\Omega)^{3}}^{2}+\|\operatorname{curl} \mathbf{G}\|_{\mathrm{L}^{2}(\Omega)^{3}}^{2}\right]^{1 / 2},
$$

and, for each positive real number $r$, let

$$
\mathrm{H}^{r}(\operatorname{curl}, \Omega):=\left\{\mathbf{G} \in \mathrm{H}^{r}(\Omega)^{3}: \operatorname{curl} \mathbf{G} \in \mathrm{H}^{r}(\Omega)^{3}\right\},
$$

endowed with the norm

$$
\|\mathbf{G}\|_{\mathrm{H}^{r}(\operatorname{curl}, \Omega)}:=\left[\|\mathbf{G}\|_{\mathrm{H}^{r}(\Omega)^{3}}^{2}+\|\mathbf{c u r l} \mathbf{G}\|_{\mathrm{H}^{r}(\Omega)^{3}}^{2}\right]^{1 / 2} .
$$


Consider the following closed subspaces of $\mathrm{H}(\operatorname{curl}, \Omega)$ :

$$
\begin{aligned}
\mathcal{V} & =\left\{\mathbf{G} \in \mathrm{H}(\operatorname{curl}, \Omega): \quad \operatorname{curl} \mathbf{G}=\mathbf{0} \text { in } \Omega_{\mathrm{D}}\right\}, \\
\mathcal{V}^{0} & =\left\{\mathbf{G} \in \mathcal{V}: \mathbf{G} \times \mathbf{n}=\mathbf{0} \text { in } \mathrm{H}_{00}^{-1 / 2}\left(\Gamma_{\mathrm{D}}\right)^{3}\right\},
\end{aligned}
$$

where $\mathrm{H}_{00}^{-1 / 2}\left(\Gamma_{\mathrm{D}}\right)^{3}$ denotes the dual space of $\mathrm{H}_{00}^{1 / 2}\left(\Gamma_{\mathrm{D}}\right)^{3}$ which, in its turn, is the space of functions defined on $\Gamma_{\mathrm{D}}$ that extended by $\mathbf{0}$ on $\partial \Omega \backslash \Gamma_{\mathrm{D}}$ belong to $\mathrm{H}^{1 / 2}(\partial \Omega)^{3}$.

We assume that $\mu, \epsilon, \sigma \in \mathrm{L}^{\infty}(\Omega)$, and that there exist constants, $\mu, \underline{\epsilon}$, and $\underline{\sigma}$, such that

$$
\begin{aligned}
\mu(\mathbf{x}) \geq \underline{\mu}>0, & \text { a.e. in } \Omega, \\
\epsilon(\mathbf{x}) \geq \underline{\epsilon}>0, & \text { a.e. in } \Omega, \\
\sigma(\mathbf{x}) \geq \underline{\sigma}>0, & \text { a.e. in } \Omega_{\mathrm{C}}, \quad \sigma(\mathbf{x})=0 \quad \text { in } \Omega_{\mathrm{D}} .
\end{aligned}
$$

Concerning the boundary data $\mathbf{f}$, we suppose there exists a field $\mathbf{H}_{\mathbf{f}} \in \mathcal{V}$ such that

$$
\mathbf{H}_{\mathbf{f}} \times \mathbf{n}=\mathbf{f} \text { in } \mathrm{H}_{00}^{-1 / 2}\left(\Gamma_{\mathrm{D}}\right)^{3} .
$$

Remark 3.1. We refer to [1] for necessary and sufficient conditions on $\mathbf{f}$ to ensure that there exists $\mathbf{H}_{\mathbf{f}} \in \mathrm{H}(\mathbf{c u r l}, \Omega)$ such that $\mathbf{H}_{\mathbf{f}} \times \mathbf{n}=\mathbf{f}$ on $\Gamma_{\mathrm{D}}$ in a weak sense, in the case $\Gamma_{\mathrm{C}}=\emptyset, \Gamma_{\mathrm{D}}=\partial \Omega$ (i.e., when the conductors $\bar{\Omega}_{\mathrm{C}}$ are fully contained in $\Omega$ ). We also refer to $[14,15]$ for similar conditions in the case that $\Omega$ is a Lipschitz polyhedron, and $\Gamma_{\mathrm{C}}$ and $\Gamma_{\mathrm{D}}$ are polyhedral surfaces with piecewise smooth boundaries.

Equation (3.1) implies an additional constraint on the data $\mathbf{f}$, since $\mathbf{H}_{\mathbf{f}}$ has to be curl-free in $\Omega_{\mathrm{D}}$. A necessary condition for the existence of such $\mathbf{H}_{\mathbf{f}}$ is that $\operatorname{div}_{\Gamma} \mathbf{f}=0$ on $\Gamma_{\mathrm{D}}$, where $\operatorname{div}_{\Gamma}$ stands for the tangential divergence operator (see [1] for the result and a precise definition of $\left.\operatorname{div}_{\Gamma}\right)$. In the case $\Gamma_{\mathrm{C}}=\emptyset, \Gamma_{\mathrm{D}}=\partial \Omega$, then $\operatorname{div}_{\Gamma} \mathbf{f}=0$ on $\Gamma_{\mathrm{D}}$ is also a sufficient condition, when $\Omega$ has a smooth boundary (see Theorem 4.1 of $[1])$.

Now, we can state a variational formulation of our problem in terms of the magnetic field $\mathbf{H}$ :

Problem MP: To find $\mathbf{H} \in \mathcal{V}$ such that

$$
\begin{aligned}
& \mathbf{H} \times \mathbf{n}=\mathbf{f} \text { in } \mathrm{H}_{00}^{-1 / 2}\left(\Gamma_{\mathrm{D}}\right)^{3}, \\
& \mathrm{i} \omega \int_{\Omega} \mu \mathbf{H} \cdot \overline{\mathbf{G}}+\int_{\Omega_{\mathrm{C}}} \frac{1}{\sigma} \operatorname{curl} \mathbf{H} \cdot \operatorname{curl} \overline{\mathbf{G}}=0 \quad \forall \mathbf{G} \in \mathcal{V}^{0} .
\end{aligned}
$$
by

Let $a: \mathrm{H}(\operatorname{curl}, \Omega) \times \mathrm{H}(\operatorname{curl}, \Omega) \longrightarrow \mathbb{C}$ be the sesquilinear continuous form defined

$$
a(\mathbf{H}, \mathbf{G}):=\mathrm{i} \omega \int_{\Omega} \mu \mathbf{H} \cdot \overline{\mathbf{G}}+\int_{\Omega_{\mathrm{C}}} \frac{1}{\sigma} \operatorname{curl} \mathbf{H} \cdot \operatorname{curl} \overline{\mathbf{G}} .
$$

This form clearly satisfies

$$
|a(\mathbf{G}, \mathbf{G})| \geq \alpha\|\mathbf{G}\|_{\mathrm{H}(\operatorname{curl}, \Omega)}^{2} \quad \forall \mathbf{G} \in \mathcal{V} .
$$

Hence, the following existence result is immediately derived: 
Theorem 3.1. If there exists $\mathbf{H}_{\mathbf{f}} \in \mathcal{V}$ such that $\mathbf{H}_{\mathbf{f}} \times \mathbf{n}=\mathbf{f}$ in $\mathrm{H}_{00}^{-1 / 2}\left(\Gamma_{\mathrm{D}}\right)^{3}$, then problem MP attains a unique solution.

Proof. Consider the translation $\hat{\mathbf{H}}=\mathbf{H}-\mathbf{H}_{\mathbf{f}}$. Then problem $\mathbf{M P}$ is equivalent to find $\hat{\mathbf{H}} \in \mathcal{V}^{0}$ such that

$$
a(\hat{\mathbf{H}}, \mathbf{G})=-a\left(\mathbf{H}_{\mathbf{f}}, \mathbf{G}\right) \quad \forall \mathbf{G} \in \mathcal{V}^{0},
$$

and this problem has a unique solution because of inequality (3.4) and Lax-Milgram Lemma.

Once the magnetic field $\mathbf{H}$ is known, the current density $\mathbf{J}$ and the electric field $\mathbf{E}$ can be readily computed in the conductors by means of equations (2.8) and (2.14), respectively. These are the magnitudes actually needed in most applications.

In the following theorem we show that the solution of problem MP satisfies some of Maxwell equations (2.8)-(2.11) and the boundary conditions (2.15)-(2.16) in a weak sense.

Theorem 3.2. Let $\mathbf{H} \in \mathcal{V}$ be the solution of problem $\mathbf{M P}$. Let $\mathbf{B}=\mu \mathbf{H} \in \mathrm{L}^{2}(\Omega)$, $\mathbf{J}=\mathbf{c u r l} \mathbf{H} \in \mathrm{L}^{2}(\Omega)$, and $\mathbf{E}=\left.\left(\frac{1}{\sigma} \mathbf{J}\right)\right|_{\Omega_{\mathrm{C}}} \in \mathrm{L}^{2}\left(\Omega_{\mathrm{C}}\right)$. Then the following properties hold true:

$$
\begin{aligned}
\operatorname{div} \mathbf{B}=0 & \text { in } \Omega, \\
i \omega \mu \mathbf{H}+\mathbf{c u r l} \mathbf{E}=\mathbf{0} & \text { in } \Omega_{\mathrm{C}} \\
\mathbf{E} \times \mathbf{n}=\mathbf{0} & \text { in } \mathrm{H}_{00}^{-1 / 2}\left(\Gamma_{\mathrm{C}}\right)^{3}, \\
\mathbf{H} \times \mathbf{n}=\mathbf{f} & \text { in } \mathrm{H}_{00}^{-1 / 2}\left(\Gamma_{\mathrm{D}}\right)^{3}, \\
\mathbf{J}=\mathbf{0} & \text { in } \Omega_{\mathrm{D}} .
\end{aligned}
$$

Proof. Given $\Psi \in \mathcal{D}(\Omega):=\left\{\Psi \in \mathcal{C}^{\infty}(\Omega): \operatorname{supp} \Psi \subset \Omega\right\}$, let $\mathbf{G}=\operatorname{grad} \Psi \in \mathcal{V}^{0}$. Then, (3.3) yields

$$
\int_{\Omega} \mu \mathbf{H} \cdot \operatorname{grad} \bar{\Psi}=0 .
$$

Consequently $\mathbf{B}=\mu \mathbf{H} \in \mathrm{H}(\operatorname{div}, \Omega)$ and (3.5) holds true.

Now, let $\mathbf{G} \in \mathcal{D}(\Omega)^{3}$ be such that $\operatorname{supp} \mathbf{G} \subset \Omega_{\mathrm{C}}$. Then $\mathbf{G} \in \mathcal{V}^{0}$ and (3.3) yields

$$
\mathrm{i} \omega \int_{\Omega_{\mathrm{C}}} \mu \mathbf{H} \cdot \overline{\mathbf{G}}+\int_{\Omega_{\mathrm{C}}} \frac{1}{\sigma} \operatorname{curl} \mathbf{H} \cdot \operatorname{curl} \overline{\mathbf{G}}=0 .
$$

Hence $\mathbf{E}=\frac{1}{\sigma} \mathbf{J}=\frac{1}{\sigma} \operatorname{curl} \mathbf{H} \in \mathrm{H}\left(\mathbf{c u r l}, \Omega_{\mathrm{C}}\right)$ and (3.6) holds true.

To prove (3.7), given $\boldsymbol{\varphi} \in \mathrm{H}_{00}^{1 / 2}\left(\Gamma_{\mathrm{C}}\right)^{3}$, we will show that $\langle\mathbf{E} \times \mathbf{n}, \tilde{\boldsymbol{\varphi}}\rangle_{\partial \Omega_{\mathrm{C}}}=0$, where $\langle\cdot, \cdot\rangle_{\partial \Omega_{\mathrm{C}}}$ denotes the duality pairing in $\mathrm{H}^{-1 / 2}\left(\partial \Omega_{\mathrm{C}}\right)^{3} \times \mathrm{H}^{1 / 2}\left(\partial \Omega_{\mathrm{C}}\right)^{3}$, and $\widetilde{\boldsymbol{\varphi}} \in \mathrm{H}^{1 / 2}\left(\partial \Omega_{\mathrm{C}}\right)^{3}$ is the natural extension of $\boldsymbol{\varphi}$ by $\mathbf{0}$ on $\partial \Omega_{\mathrm{C}} \backslash \Gamma_{\mathrm{C}}$. To this aim, let $\mathbf{G} \in \mathrm{H}^{1}\left(\Omega_{\mathrm{C}}\right)^{3}$ be such that $\left.\mathbf{G}\right|_{\partial \Omega_{\mathrm{C}}}=\widetilde{\varphi}$ and $\widetilde{\mathbf{G}}$ be the extension by $\mathbf{0}$ of $\mathbf{G}$ to $\Omega \backslash \Omega_{\mathrm{C}}$. Then $\widetilde{\mathbf{G}} \in \mathcal{V}^{0}$ and (3.3) yields

$$
\begin{aligned}
0 & =\mathrm{i} \omega \int_{\Omega} \mu \mathbf{H} \cdot \overline{\widetilde{\mathbf{G}}}+\int_{\Omega_{\mathrm{C}}} \mathbf{E} \cdot \operatorname{curl} \overline{\widetilde{\mathbf{G}}} \\
& =\mathrm{i} \omega \int_{\Omega_{\mathrm{C}}} \mu \mathbf{H} \cdot \overline{\mathbf{G}}+\int_{\Omega_{\mathrm{C}}} \operatorname{curl} \mathbf{E} \cdot \overline{\mathbf{G}}+\left\langle\mathbf{E} \times \mathbf{n},\left.\mathbf{G}\right|_{\partial \Omega_{\mathrm{C}}}\right\rangle_{\partial \Omega_{\mathrm{C}}}=\langle\mathbf{E} \times \mathbf{n}, \widetilde{\varphi}\rangle_{\partial \Omega_{\mathrm{C}}}
\end{aligned}
$$


where we have used that $\mathbf{E}=\frac{1}{\sigma} \operatorname{curl} \mathbf{H}$ in $\Omega_{\mathrm{C}}$ and (3.6).

Finally, (3.8) and (3.9) arise explicitly in problem MP.

Remark 3.2. The theorem above shows that problem MP allows us to determine uniquely the electric field $\mathbf{E}$ in the conductors. In its turn, $\mathbf{E}$ and Maxwell equation (2.11) determines the charge density $\rho$ in $\Omega_{\mathrm{C}}$. In particular, in the interior of any homogeneous subdomain $\Omega^{\prime}$ of $\Omega_{\mathrm{C}}$ (i.e., $\Omega^{\prime} \subset \Omega_{\mathrm{C}}$ such that $\left.\epsilon\right|_{\Omega^{\prime}}$ and $\left.\sigma\right|_{\Omega^{\prime}}$ are constant), $\left.\rho\right|_{\Omega^{\prime}}=\left.\operatorname{div}\left(\frac{\epsilon}{\sigma} \operatorname{curl} \mathbf{H}\right)\right|_{\Omega^{\prime}}=0$.

Instead, the electric field $\mathbf{E}$ is not uniquely determined in the dielectric. Indeed, from the eddy currents model (2.8)-(2.14) we obtain the following equations for $\left.\mathbf{E}\right|_{\Omega_{\mathrm{D}}}$ :

$$
\begin{aligned}
\operatorname{curl} \mathbf{E}=-\mathrm{i} \omega \mu \mathbf{H} & \text { in } \Omega_{\mathrm{D}}, \\
\operatorname{div}(\epsilon \mathbf{E})=\rho & \text { in } \Omega_{\mathrm{D}}, \\
\mathbf{E} \times \mathbf{n}=\left.\mathbf{E}\right|_{\Omega_{\mathrm{C}}} \times \mathbf{n} & \text { on } \Gamma_{\mathrm{I}} .
\end{aligned}
$$

The latter arises from the facts that $\left.\mathbf{E}\right|_{\Omega_{\mathrm{C}}}$ is already known and $\mathbf{E}$ is globally in $\mathrm{H}(\operatorname{curl}, \Omega)$.

A boundary condition on $\Gamma_{\mathrm{D}}$ is needed to determine a unique solution, even in the simplest case of a topologically trivial $\Omega_{\mathrm{D}}$ (i.e., when $\Omega_{\mathrm{D}}$ is simply connected with a connected boundary). A natural condition would be to impose the normal component of the electric displacement $\mathbf{D}$ on $\Gamma_{\mathrm{D}}$; namely,

$$
\epsilon \mathbf{E} \cdot \mathbf{n}=\psi \quad \text { on } \Gamma_{\mathrm{D}} .
$$

The data $\psi$ amounts to eventual surface charges on the outer boundary of the dielectric domain.

Existence of solution of (3.10)-(3.13) has been proved in Theorem 4.2 of [1] in the case that $\partial \Omega_{\mathrm{D}}$ is smooth and $\Gamma_{\mathrm{I}} \cap \Gamma_{\mathrm{D}}=\emptyset$ (for instance, when $\bar{\Omega}_{\mathrm{C}} \subset \Omega$ ). Even in this simpler case, a number of additional constraints related with the topology of $\Omega_{\mathrm{D}}$ must be added to have uniqueness, as can be seen in this reference.

To the best of the authors' knowledge, a similar result has not been proved for the general case of $\Omega_{\mathrm{D}}$ being a Lipschitz polyhedron with $\Gamma_{\mathrm{I}} \cap \Gamma_{\mathrm{D}} \neq \emptyset$. Nevertheless, this is not a drawback for the application of this eddy currents model, since typically the goal of these problems is to compute the electric field only in the conductors, as said above.

4. Introducing a magnetic potential. In this section we show how problem MP can be transformed by replacing the magnetic field in the dielectric domain $\Omega_{\mathrm{D}}$ by a (scalar) magnetic potential.

We recall that $\Omega$ is assumed to be simply connected with connected boundary $\partial \Omega$. Let $\Omega_{\mathrm{C}}=\bigcup_{j=0}^{j=J} \Omega_{\mathrm{C}}^{j}$, with $\Omega_{\mathrm{C}}^{0}$ being the union of all the connected components of $\Omega_{\mathrm{C}}$ such that $\Omega \backslash \Omega_{\mathrm{C}}^{0}$ is simply connected, and $\Omega_{\mathrm{C}}^{j}, j=1, \ldots, J$, the remaining connected components of $\Omega_{\mathrm{C}}$ (see Figure 4.1).

We assume that for each $\Omega_{\mathrm{C}}^{j}, j=1, \ldots, J$, there exists an open "cut" surface $\Sigma_{j} \subset \Omega_{\mathrm{D}}$ such that $\partial \Sigma_{j} \subset \partial \Omega_{\mathrm{D}}$ and $\widetilde{\Omega}_{\mathrm{D}}:=\Omega_{\mathrm{D}} \backslash \bigcup_{j=0}^{j=J} \bar{\Sigma}_{j}$ is pseudo-Lipschitz and simply connected (see Figure 4.1). We also assume that each one of these surfaces $\Sigma_{j}$ is connected, and $\bar{\Sigma}_{j} \cap \bar{\Sigma}_{k}=\emptyset$ for $j \neq k$ (see, for instance, [5]).

Let us arrange the conductors $\Omega_{\mathrm{C}}^{j}$ in such a way that the inner ones are numbered from $j=1$ to $K$, and those going through $\partial \Omega$, from $j=K+1$ to $J$. In Figure 4.1, $\Omega_{\mathrm{C}}^{1}$ is an example of a conductor of the first kind, and $\Omega_{\mathrm{C}}^{2}$ of the second one. 


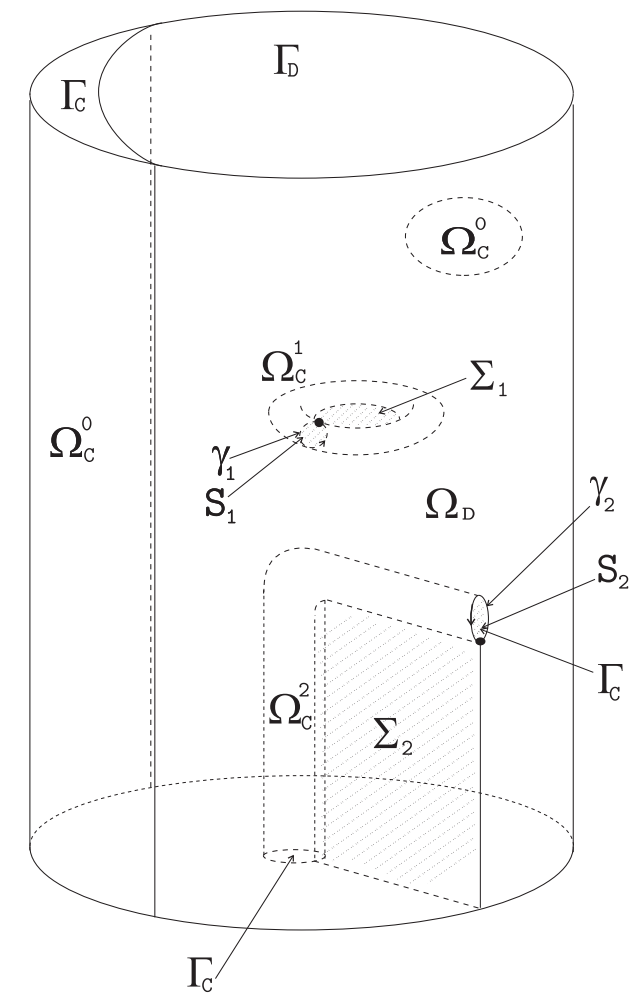

FIG. 4.1. Sketch of the domain.

We also assume that there exist cross sections of $\Omega_{\mathrm{C}}^{j}, j=1 \ldots J$; namely, open surfaces $S_{j} \subset \bar{\Omega}_{\mathrm{C}}^{j}$, with respective boundaries $\partial S_{j}=\bar{S}_{j} \cap \Gamma_{\mathrm{I}}$, which are assumed to be closed simple curves. We denote these curves $\gamma_{j}$. Moreover, for $j=K+1, \ldots, J$, we take $S_{j} \subset \Gamma_{\mathrm{C}}$ and $\gamma_{j} \subset \Gamma_{\mathrm{C}} \cap \Gamma_{\mathrm{D}}$ (see again Figure 4.1).

Let $\widetilde{\Omega}_{\mathrm{D}}^{j}:=\Omega_{\mathrm{D}} \backslash \bar{\Sigma}_{j}, j=1 \ldots J$. We fix a unit normal $\mathbf{n}_{j}$ on each $\Sigma_{j}$ and denote its two faces $\Sigma_{j}^{-}$and $\Sigma_{j}^{+}$, with $\mathbf{n}_{j}$ being the "outer" normal to $\widetilde{\Omega}_{\mathrm{D}}^{j}$ along $\Sigma_{j}^{+}$. We choose an orientation for each $\gamma_{j}$ by taking its initial and end points on $\bar{\Sigma}_{j}^{-}$and $\bar{\Sigma}_{j}^{+}$, respectively. We denote by $\mathbf{t}_{j}$, the unit vector tangent to $\gamma_{j}$.

For any function $\widetilde{\Psi} \in \mathrm{H}^{1}\left(\widetilde{\Omega}_{\mathrm{D}}\right)$, we denote by

$$
\llbracket \widetilde{\Psi} \rrbracket_{\Sigma_{j}}:=\left.\widetilde{\Psi}\right|_{\Sigma_{j}^{-}}-\left.\widetilde{\Psi}\right|_{\Sigma_{j}^{+}}
$$

the jump of $\widetilde{\Psi}$ through $\Sigma_{j}$ along $\mathbf{n}_{j}$. The gradient of $\widetilde{\Psi}$ in $\mathcal{D}^{\prime}\left(\widetilde{\Omega}_{\mathrm{D}}\right)$ can be extended to $\mathrm{L}^{2}\left(\Omega_{\mathrm{D}}\right)^{3}$ and will be denoted by $\operatorname{grad} \widetilde{\Psi}$.

Let $\Theta$ be the linear space of $\mathrm{H}^{1}\left(\widetilde{\Omega}_{\mathrm{D}}\right)$ defined by

$$
\Theta=\left\{\widetilde{\Psi} \in \mathrm{H}^{1}\left(\widetilde{\Omega}_{\mathrm{D}}\right): \llbracket \widetilde{\Psi} \rrbracket_{\Sigma_{j}}=\text { constant }, j=1, \ldots, J\right\} .
$$

Then, for $\widetilde{\Psi} \in \mathrm{H}^{1}\left(\widetilde{\Omega}_{\mathrm{D}}\right)$, we have that $\operatorname{grad} \widetilde{\Psi} \in \mathrm{H}\left(\operatorname{curl}, \Omega_{\mathrm{D}}\right)$ if and only if $\widetilde{\Psi} \in \Theta$, in which case $\operatorname{curl}(\operatorname{grad} \widetilde{\Psi})=\mathbf{0}$ (see Lemma 3.11 in [5]). Actually, the kernel of the operator curl : $\mathrm{H}\left(\operatorname{curl}, \Omega_{\mathrm{D}}\right) \longrightarrow \mathrm{L}^{2}\left(\Omega_{\mathrm{D}}\right)^{3}$ is given by

$$
\operatorname{Ker}(\operatorname{curl})=\operatorname{grad} \Theta=\operatorname{grad}^{1}\left(\Omega_{\mathrm{D}}\right) \oplus \mathcal{C},
$$


where $\mathcal{C}$ is the space of the so-called Neumann harmonic fields in $\Omega_{\mathrm{D}}$ defined by

$$
\mathcal{C}:=\left\{\mathbf{G} \in \mathrm{L}^{2}\left(\Omega_{\mathrm{D}}\right)^{3}: \operatorname{curl} \mathbf{G}=\mathbf{0}, \operatorname{div}(\mu \mathbf{G})=0 \text { in } \Omega_{\mathrm{D}}, \text { and } \mathbf{G} \cdot \mathbf{n}=\mathbf{0} \text { on } \partial \Omega_{\mathrm{D}}\right\} .
$$

A basis of the space $\mathcal{C}$ is given by the set of functions $\left\{\boldsymbol{\operatorname { g r a d }} \widetilde{\Phi}_{j}, j=1, \ldots, J\right\}$, where, for each $j, \widetilde{\Phi}_{j} \in \mathrm{H}^{1}\left(\widetilde{\Omega}_{\mathrm{D}}^{j}\right)$ is the solution of

$$
\begin{aligned}
& \int_{\widetilde{\Omega}_{\mathrm{D}}^{j}} \mu \operatorname{grad} \widetilde{\Phi}_{j} \cdot \operatorname{grad} \bar{\Psi}=0 \quad \forall \Psi \in \mathrm{H}^{1}\left(\Omega_{\mathrm{D}}\right), \\
& \llbracket \widetilde{\Phi}_{j} \rrbracket_{\Sigma_{j}}=1 .
\end{aligned}
$$

By using Lax-Milgram Lemma, it is straightforward to see that $\widetilde{\Phi}_{j}$ is uniquely defined in $\mathrm{H}^{1}\left(\widetilde{\Omega}_{\mathrm{D}}^{j}\right) / \mathbb{C}$. (See, for instance, again [5].)

Therefore, according to (4.1), for all $\mathbf{G} \in \mathcal{V}$, there exist unique constants $c_{j}$, $j=1, \ldots, J$, and a unique scalar field $\Psi \in \mathrm{H}^{1}\left(\Omega_{\mathrm{D}}\right) / \mathbb{C}$, such that $\left.\mathbf{G}\right|_{\Omega_{\mathrm{D}}}=\operatorname{grad} \widetilde{\Psi}$, with $\widetilde{\Psi} \in \Theta$ given by $\widetilde{\Psi}=\Psi+\sum_{j=1}^{J} c_{j} \widetilde{\Phi}_{j}$. Furthermore, because of (4.3), the constants $c_{j}$ are the jumps of $\widetilde{\Psi}$ across the respective cuts $\Sigma_{j}$. Consequently, given $\widetilde{\Psi} \in \Theta$, we have that $\widetilde{\Psi} \in \mathrm{H}^{1}(\Omega)$ if and only if $\llbracket \widetilde{\Psi} \rrbracket_{\Sigma_{j}}=0$ for $j=1, \ldots, J$.

Remark 4.1. These jumps have a precise physical meaning. For instance, for the solution $\mathbf{H}$ of problem $\mathbf{M P}$, let us write $\left.\mathbf{H}\right|_{\Omega_{\mathrm{D}}}=\operatorname{grad} \widetilde{\Phi}$ with $\widetilde{\Phi} \in \Theta$. If $\mathbf{H}$ is sufficiently smooth, by using Stokes theorem and equation (2.8) we have

$$
\begin{aligned}
\llbracket \widetilde{\Phi} \rrbracket_{\Sigma_{j}} & =\int_{\gamma_{j}} \operatorname{grad} \widetilde{\Phi} \cdot \mathbf{t}_{j} d \gamma=\left.\int_{\gamma_{j}} \mathbf{H}\right|_{\Omega_{\mathrm{D}}} \cdot \mathbf{t}_{j} d \gamma=\left.\int_{\gamma_{j}} \mathbf{H}\right|_{\Omega_{\mathrm{C}}} \cdot \mathbf{t}_{j} d \gamma \\
& =\left.\int_{S_{j}} \operatorname{curl} \mathbf{H}\right|_{\Omega_{\mathrm{C}}} \cdot \mathbf{n} d \Gamma=\int_{S_{j}} \mathbf{J} \cdot \mathbf{n} d \Gamma=: I_{j}, \quad j=1, \ldots, J .
\end{aligned}
$$

Thus, the jump of the magnetic potential $\widetilde{\Psi}$ across each cut surface $\Sigma_{j}$ is exactly the current intensity $I_{j}$ through the cross section $S_{j}$ of the conductor $\Omega_{\mathrm{C}}^{j}$ (as defined above).

We introduce the following notation: for $\mathbf{G}_{\mathrm{C}} \in \mathrm{L}^{2}\left(\Omega_{\mathrm{C}}\right)^{3}$ and $\mathbf{G}_{\mathrm{D}} \in \mathrm{L}^{2}\left(\Omega_{\mathrm{D}}\right)^{3}$, we denote by $\left(\mathbf{G}_{\mathrm{C}} \mid \mathbf{G}_{\mathrm{D}}\right)$ the field $\mathbf{G} \in \mathrm{L}^{2}(\Omega)^{3}$ defined a.e. by

$$
\mathbf{G}(\mathbf{x}):= \begin{cases}\mathbf{G}_{\mathrm{C}}(\mathbf{x}) & \text { if } \mathbf{x} \in \Omega_{\mathrm{C}} \\ \mathbf{G}_{\mathrm{D}}(\mathbf{x}) & \text { if } \mathbf{x} \in \Omega_{\mathrm{D}}\end{cases}
$$

Let us denote by $\mathcal{W}$ the linear space given by

$$
\mathcal{W}:=\left\{(\mathbf{G}, \widetilde{\Psi}) \in \mathrm{H}\left(\mathbf{c u r l}, \Omega_{\mathrm{C}}\right) \times(\Theta / \mathbb{C}):(\mathbf{G} \mid \operatorname{grad} \widetilde{\Psi}) \in \mathrm{H}(\operatorname{curl}, \Omega)\right\} .
$$

Clearly, the following application is an isomorphism:

$$
\begin{array}{ccc}
\mathcal{W} & \longrightarrow & \mathcal{V} \\
(\mathbf{G}, \widetilde{\Psi}) & \longmapsto & (\mathbf{G} \mid \operatorname{grad} \widetilde{\Psi})
\end{array}
$$

Similarly, we define the closed subspace of $\mathcal{W}$

$$
\mathcal{W}^{0}:=\left\{(\mathbf{G}, \widetilde{\Psi}) \in \mathcal{W}: \operatorname{grad} \widetilde{\Psi} \times \mathbf{n}=\mathbf{0} \text { in } \mathrm{H}_{00}^{-1 / 2}\left(\Gamma_{\mathrm{D}}\right)^{3}\right\}
$$


which is isomorphically equivalent to $\mathcal{V}^{0}$.

Thus, we are lead to define the following problem:

Problem HP: To find $(\mathbf{H}, \widetilde{\Phi}) \in \mathcal{W}$ such that

$$
\begin{array}{r}
\operatorname{grad} \widetilde{\Phi} \times \mathbf{n}=\mathbf{f} \text { in } \mathrm{H}_{00}^{-1 / 2}\left(\Gamma_{\mathrm{D}}\right)^{3}, \\
\mathrm{i} \omega \int_{\Omega_{\mathrm{C}}} \mu \mathbf{H} \cdot \overline{\mathbf{G}}+\int_{\Omega_{\mathrm{C}}} \frac{1}{\sigma} \operatorname{curl} \mathbf{H} \cdot \operatorname{curl} \overline{\mathbf{G}}+\mathrm{i} \omega \int_{\Omega_{\mathrm{D}}} \mu \mathbf{g r a d} \widetilde{\Phi} \cdot \operatorname{grad} \overline{\widetilde{\Psi}}=0 \\
\forall(\mathbf{G}, \widetilde{\Psi}) \in \mathcal{W}^{0} .
\end{array}
$$

This is the well known magnetic field/magnetic potential hybrid formulation of the eddy currents problem introduced by Bossavit and Verité [13]. One main advantage with respect to formulation (3.2)-(3.3) lies in the fact that a vector field is replaced by a scalar one in the dielectric domain.

The following lemma is an immediate consequence of the isomorphisms between $\mathcal{W}$ and $\mathcal{V}$, and between $\mathcal{W}^{0}$ and $\mathcal{V}^{0}$ :

Lemma 4.1. The pair $(\mathbf{H}, \widetilde{\Phi})$ is solution of problem $\mathbf{H P}$ if and only if $(\mathbf{H} \mid \operatorname{grad} \widetilde{\Phi})$ is solution of problem MP.

As a consequence of this lemma, Theorem 3.1 yields existence and uniqueness of solution for problem HP:

COROLlary 4.2. Under the assumptions of Theorem 3.1, problem HP has a unique solution $(\mathbf{H}, \widetilde{\Phi})$, with $(\mathbf{H} \mid \operatorname{grad} \widetilde{\Phi})$ being the unique solution of problem $\mathbf{M P}$.

5. Numerical solution. In this section we first introduce a discretization of problem MP and prove its convergence. Then we prove that the obtained discrete problem is completely equivalent to a convenient discrete version of problem HP.

5.1. Discretizing the magnetic field. We employ "edge" finite elements to approximate the magnetic field; more precisely, the lowest-order finite element of the family introduced by Nédélec in [26]. This element belongs to the family of the so-called Whitney elements (see [9]).

We assume $\Omega, \Omega_{\mathrm{C}}$, and $\Omega_{\mathrm{D}}$ are Lipschitz polyhedra, and consider a family of regular tetrahedral meshes $\left\{\mathcal{T}_{h}\right\}$ of $\Omega$ such that, for every mesh $\mathcal{T}_{h}$, each element $K \in \mathcal{T}_{h}$ is contained either in $\bar{\Omega}_{\mathrm{C}}$ or in $\bar{\Omega}_{\mathrm{D}}$ ( $h$ stands as usual for the corresponding mesh-size).

The magnetic field is approximated in each tetrahedron $K$ by a polynomial vector field in the space

$$
\mathcal{N}(K):=\left\{\mathbf{G}_{h} \in \mathcal{P}_{1}(K)^{3}: \mathbf{G}_{h}(\mathbf{x})=\mathbf{a} \times \mathbf{x}+\mathbf{b}, \mathbf{a}, \mathbf{b} \in \mathbb{C}^{3}, \mathbf{x} \in K\right\} .
$$

An explicit computation shows that vector fields of this type have constant tangential components along each straight line in the Euclidean space. Moreover, given six complex numbers $\beta_{n}, n=1, \ldots, 6$, there exists a unique $\mathbf{G}_{h} \in \mathcal{N}(K)$ (i.e., unique $\mathbf{a}, \mathbf{b} \in \mathbb{C}^{3}$ ) such that its tangential component along the $n$-th edge of $K$ coincide with $\beta_{n}$, for $n=1 \ldots 6$, respectively. Thus, these tangential components along the edges of $K$ can be taken as the degrees of freedom defining the elements in $\mathcal{N}(K)$.

These elements are $\mathrm{H}(\mathbf{c u r l})$-conforming in the sense that, $\forall \mathbf{G}_{h} \in \mathcal{N}(K)$, their tangential traces on each triangular face $T$ of $K$ only depend on the degrees of freedom of $\mathbf{G}_{h}$ on the three edges of $T$. So, if we set

$$
\mathcal{N}_{h}(\Omega):=\left\{\mathbf{G}_{h} \in \mathrm{H}(\operatorname{curl}, \Omega):\left.\mathbf{G}_{h}\right|_{K} \in \mathcal{N}(K) \forall K \in \mathcal{T}_{h}\right\},
$$


the elements in this space are piecewise linear vector fields with tangential traces that are continuous through the faces of the mesh. This is the lowest-order Nédélec finite element space introduced in [26]. See [19] for a detailed mathematical analysis and [11] for useful implementation issues.

If $\mathbf{G}$ is smooth enough (v.g., $\mathbf{G} \in \mathrm{H}^{2}(\Omega)^{3}$ ), then its Nédélec interpolant $\mathbf{G}^{\mathrm{I}}$ is defined by

$$
\mathbf{G}^{\mathrm{I}} \in \mathcal{N}_{h}(\Omega): \quad \int_{\ell} \mathbf{G}^{\mathrm{I}} \cdot \mathbf{t}_{\ell} d \gamma=\int_{\ell} \mathbf{G} \cdot \mathbf{t}_{\ell} d \gamma \quad \forall \ell \text { edge of } \mathcal{T}_{h},
$$

where, from now on, $\mathbf{t}_{\ell}$ denotes a unit vector tangent to the edge $\ell$. The Nédélec interpolation operator

$$
\begin{aligned}
\mathrm{H}^{2}(\Omega)^{3} & \longrightarrow \mathcal{N}_{h}(\Omega) \\
\mathbf{G} & \longmapsto \mathbf{G}^{\mathrm{I}}
\end{aligned}
$$

with $\mathbf{G}^{\mathrm{I}}$ defined by (5.1), extends uniquely to $\mathrm{H}^{r}$ (curl, $\Omega$ ) with $r>1 / 2$. Indeed, according to Sobolev Imbedding Theorem and a trace theorem, for each $K \in \mathcal{T}_{h}$, $\left.\mathbf{G}\right|_{K} \in \mathrm{L}^{p}(K)^{3},\left.\operatorname{curl} \mathbf{G}\right|_{K} \in \mathrm{L}^{p}(K)^{3}$, and $\mathbf{G} \times\left.\mathbf{n}\right|_{\partial K} \in \mathrm{L}^{p}(\partial K)^{3}$, with $p=4 /(3-2 r)>$ 2. Then, the result follows by applying Lemma 4.7 of [5].

However, the solution $\mathbf{H}$ of problem $\mathbf{M P}$ does not satisfy, in general, curl $\mathbf{H}=$ $\mathbf{J} \in \mathrm{H}^{r}(\Omega)^{3}$ with $r>1 / 2$. In fact, $\left.\mathbf{J}\right|_{\Omega_{\mathrm{D}}}=0$, whereas $\left.\mathbf{J}\right|_{\Omega_{\mathrm{C}}} \times \mathbf{n}=\left.\left(\frac{1}{\sigma} \mathbf{E}\right)\right|_{\Omega_{\mathrm{C}}} \times \mathbf{n}$ in general does not vanish on $\Gamma_{\mathrm{I}}$; thus, $\mathbf{J} \times \mathbf{n}$ has a jump across $\Gamma_{\mathrm{I}}$ (see, for instance, the problem in Section 7). Nevertheless, typically $\left.\mathbf{H}\right|_{\Omega_{\mathrm{C}}} \in \mathrm{H}^{r}\left(\mathbf{c u r l}, \Omega_{\mathrm{C}}\right)$ and $\left.\mathbf{H}\right|_{\Omega_{\mathrm{D}}} \in \mathrm{H}^{r}\left(\mathbf{c u r l}, \Omega_{\mathrm{D}}\right)$ with $r>1 / 2$. This is enough for $\mathbf{H}^{\mathrm{I}}$ to be well defined as shown in the following Lemma, which also provides an error estimate for the Nédélec interpolant under these assumptions. (Here and thereafter $C$ denotes a generic constant, not necessarily the same at each occurrence, but always independent of the mesh-size $h$.)

Lemma 5.1. Let $r \in\left(\frac{1}{2}, 1\right]$. The operator defined by (5.2)-(5.1) extends uniquely to the space $\left\{\mathbf{G} \in \mathrm{H}(\mathbf{c u r l}, \Omega):\left.\mathbf{G}\right|_{\Omega_{\mathrm{C}}} \in \mathrm{H}^{r}\left(\mathbf{c u r l}, \Omega_{\mathrm{C}}\right)\right.$ and $\left.\left.\mathbf{G}\right|_{\Omega_{\mathrm{D}}} \in \mathrm{H}^{r}\left(\mathbf{c u r l}, \Omega_{\mathrm{D}}\right)\right\}$. Furthermore, for all $\mathbf{G}$ in this space,

$$
\left\|\mathbf{G}-\mathbf{G}^{\mathrm{I}}\right\|_{\mathrm{H}(\operatorname{curl}, \Omega)} \leq C h^{r}\left[\|\mathbf{G}\|_{\mathrm{H}^{r}\left(\operatorname{curl}, \Omega_{\mathrm{C}}\right)}+\|\mathbf{G}\|_{\mathrm{H}^{r}\left(\operatorname{curl}, \Omega_{\mathrm{D}}\right)}\right]
$$

Proof. According to the discussion above, since $\left.\mathbf{G}\right|_{\Omega_{\mathrm{C}}} \in \mathrm{H}^{r}\left(\operatorname{curl}, \Omega_{\mathrm{C}}\right)$ and $\left.\mathbf{G}\right|_{\Omega_{\mathrm{D}}} \in$ $\mathrm{H}^{r}\left(\mathbf{c u r l}, \Omega_{\mathrm{D}}\right)$, with $r>1 / 2$, then the Nédélec interpolants of $\left.\mathbf{G}\right|_{\Omega_{\mathrm{C}}}$ and $\left.\mathbf{G}\right|_{\Omega_{\mathrm{D}}}$ are well defined in $\mathcal{N}_{h}\left(\Omega_{\mathrm{C}}\right)$ and $\mathcal{N}_{h}\left(\Omega_{\mathrm{D}}\right)$, respectively. Moreover, since $\mathbf{G} \in \mathrm{H}(\mathbf{c u r l}, \Omega)$, a density argument shows that the degrees of freedom corresponding to the edges $\ell \subset \Gamma_{\mathrm{I}}$ coincide for both interpolants. Thus the global interpolant $\mathbf{G}^{\mathrm{I}} \in \mathcal{N}_{h}(\Omega)$ is well defined also in this case.

On the other hand, the arguments in the proof of Theorem 5.4 in [19] can be extended to this case to prove the error estimate above.

In order to use these elements to discretize problem $\mathbf{M P}$, we have to use an approximant $\mathbf{f}_{\mathrm{I}}$ of the boundary data $\mathbf{f}$ such that a discrete version of equation (3.2) can hold true, namely, such that there exists $\mathbf{H}_{h} \in \mathcal{N}_{h}(\Omega)$ satisfying $\mathbf{H}_{h} \times \mathbf{n}=\mathbf{f}_{\mathrm{I}}$.

To attain this goal, we will use the two-dimensional Nédélec interpolant of $\mathbf{n} \times \mathbf{f}$ on the triangular mesh induced by $\mathcal{T}_{h}$ on the polyhedral surface $\Gamma_{\mathrm{D}}$. To introduce this interpolant, let $\mathcal{T}_{h}^{\Gamma_{\mathrm{D}}}:=\left\{T \subset \Gamma_{\mathrm{D}}: T\right.$ face of $\left.K \in \mathcal{T}_{h}\right\}$. For each triangle $T \in \mathcal{T}_{h}^{\Gamma_{\mathrm{D}}}$, 
consider local orthogonal coordinates $(\xi, \eta, \zeta)$ such that $T$ is contained in the plane $\zeta=0$. Let

$\mathcal{N}^{2}(T):=\left\{\boldsymbol{\varphi}_{h} \in \mathcal{P}_{1}(T)^{3}: \boldsymbol{\varphi}_{h}(\xi, \eta, 0)=(a-c \eta, b+c \xi, 0), a, b, c \in \mathbb{C},(\xi, \eta, 0) \in T\right\}$.

This is the lowest-order two-dimensional Nédélec finite element (see [26]) on the plane $\zeta=0$. The tangential components of these vector fields along the three edges of the triangle $T$ can also be taken as the degrees of freedom defining them. Therefore, we define

$$
\begin{aligned}
\mathcal{N}_{h}^{2}\left(\Gamma_{\mathrm{D}}\right):=\left\{\boldsymbol{\varphi}_{h} \in \mathrm{L}^{2}\left(\Gamma_{\mathrm{D}}\right)^{3}:\right. & \left.\boldsymbol{\varphi}_{h}\right|_{T} \in \mathcal{N}^{2}(T) \forall T \in \mathcal{T}_{h}^{\Gamma_{\mathrm{D}}} \\
& \text { and } \left.\boldsymbol{\varphi}_{h} \cdot \mathbf{t}_{\ell} \text { is continuous on } \ell \forall \ell \text { edge of } \mathcal{T}_{h}^{\Gamma_{\mathrm{D}}}\right\} .
\end{aligned}
$$

Let $\boldsymbol{\varphi}$ be a tangential vector field on $\Gamma_{\mathrm{D}}$ (i.e., satisfying $\boldsymbol{\varphi} \cdot \mathbf{n}=0$ on $\Gamma_{\mathrm{D}}$ ). If $\boldsymbol{\varphi}$ is sufficiently smooth (v.g., $\left.\varphi \in \mathrm{H}^{1}\left(\Gamma_{\mathrm{D}}\right)^{3}\right)$, then its Nédélec interpolant on $\Gamma_{\mathrm{D}}$, which we denote by $\varphi^{I_{2}}$, is defined by

$$
\varphi^{\mathrm{I}_{2}} \in \mathcal{N}_{h}^{2}\left(\Gamma_{\mathrm{D}}\right): \quad \int_{\ell} \boldsymbol{\varphi}^{\mathrm{I}_{2}} \cdot \mathbf{t}_{\ell} d \gamma=\int_{\ell} \boldsymbol{\varphi} \cdot \mathbf{t}_{\ell} d \gamma \quad \forall \ell \text { edge of } \mathcal{T}_{h}^{\Gamma_{\mathrm{D}}} .
$$

If $\mathbf{G}$ is smooth enough in $\Omega_{\mathrm{D}}$ (v.g., $\left.\mathbf{G} \in \mathrm{H}^{2}\left(\Omega_{\mathrm{D}}\right)^{3}\right)$, then its tangential trace on $\Gamma_{\mathrm{D}}$, $\mathbf{n} \times\left(\left.\mathbf{G}\right|_{\Gamma_{\mathrm{D}}} \times \mathbf{n}\right)$, is smooth too and satisfies

$$
\left[\mathbf{n} \times\left(\left.\mathbf{G}\right|_{\Gamma_{\mathrm{D}}} \times \mathbf{n}\right)\right]^{\mathrm{I}_{2}}=\mathbf{n} \times\left(\left.\mathbf{G}^{\mathrm{I}}\right|_{\Gamma_{\mathrm{D}}} \times \mathbf{n}\right) \quad \text { on } \Gamma_{\mathrm{D}} .
$$

Indeed, a straightforward computation shows that the right hand side above also belongs to $\mathcal{N}_{h}^{2}\left(\Gamma_{\mathrm{D}}\right)$. On the other hand, (5.1) implies

$$
\begin{aligned}
\int_{\ell} \mathbf{n} \times\left(\left.\mathbf{G}^{\mathrm{I}}\right|_{\Gamma_{\mathrm{D}}} \times \mathbf{n}\right) \cdot \mathbf{t}_{\ell} d \gamma & =\int_{\ell} \mathbf{G}^{\mathrm{I}} \cdot \mathbf{t}_{\ell} d \gamma=\int_{\ell} \mathbf{G} \cdot \mathbf{t}_{\ell} d \gamma \\
& =\int_{\ell} \mathbf{n} \times\left(\left.\mathbf{G}\right|_{\Gamma_{\mathrm{D}}} \times \mathbf{n}\right) \cdot \mathbf{t}_{\ell} d \gamma \quad \forall \ell \text { edge of } \mathcal{T}_{h}^{\Gamma_{\mathrm{D}}} .
\end{aligned}
$$

Thus, the degrees of freedom defining both sides of (5.4) coincide and, consequently, (5.4) holds true.

The following lemma shows that a similar result is valid for $\mathbf{G} \in \mathrm{H}^{r}\left(\operatorname{curl}, \Omega_{\mathrm{D}}\right)$ :

Lemma 5.2. Let $r \in\left(\frac{1}{2}, 1\right]$. The linear operator

$$
\begin{aligned}
\mathrm{H}^{2}\left(\Omega_{\mathrm{D}}\right)^{3} & \longrightarrow \mathcal{N}_{h}^{2}\left(\Gamma_{\mathrm{D}}\right) \\
\mathbf{G} & \longmapsto\left[\mathbf{n} \times\left(\left.\mathbf{G}\right|_{\Gamma_{\mathrm{D}}} \times \mathbf{n}\right)\right]^{\mathrm{I}_{2}}
\end{aligned}
$$

with $(\cdot)^{\mathrm{I}_{2}}$ defined by (5.3), extends uniquely to $\mathrm{H}^{r}\left(\mathbf{c u r l}, \Omega_{\mathrm{D}}\right)$. Furthermore, equation (5.4) holds true for all $\mathbf{G}$ in this space.

Proof. As said above, if $\mathbf{G} \in \mathrm{H}^{2}\left(\Omega_{\mathrm{D}}\right)^{3}$, then $\left[\mathbf{n} \times\left(\left.\mathbf{G}\right|_{\Gamma_{\mathrm{D}}} \times \mathbf{n}\right)\right]^{\mathrm{I}_{2}} \in \mathcal{N}_{h}^{2}\left(\Gamma_{\mathrm{D}}\right)$ is defined by

$$
\int_{\ell}\left[\mathbf{n} \times\left(\left.\mathbf{G}\right|_{\Gamma_{\mathrm{D}}} \times \mathbf{n}\right)\right]^{\mathrm{I}_{2}} \cdot \mathbf{t}_{\ell} d \gamma=\int_{\ell} \mathbf{G} \cdot \mathbf{t}_{\ell} d \gamma \quad \forall \ell \text { edge of } \mathcal{T}_{h}^{\Gamma_{\mathrm{D}}} .
$$

Then, by repeating the arguments in the proof of Lemma 5.1 (i.e., using Sobolev Imbedding Theorem and Lemma 4.7 of [5]) we prove that the operator defined by (5.5) and (5.3) extends uniquely to $\mathrm{H}^{r}\left(\mathbf{c u r l}, \Omega_{\mathrm{D}}\right)$, for $r>1 / 2$. 
Furthermore, we have also shown above that, for $\mathbf{G} \in \mathrm{H}^{2}\left(\Omega_{\mathrm{D}}\right)$,

$$
\begin{aligned}
\int_{\ell}\left[\mathbf{n} \times\left(\left.\mathbf{G}\right|_{\Gamma_{\mathrm{D}}} \times \mathbf{n}\right)\right]^{\mathrm{I}_{2}} \cdot \mathbf{t}_{\ell} d \gamma & =\int_{\ell} \mathbf{G} \cdot \mathbf{t}_{\ell} d \gamma \\
& =\int_{\ell} \mathbf{n} \times\left(\left.\mathbf{G}^{\mathrm{I}}\right|_{\Gamma_{\mathrm{D}}} \times \mathbf{n}\right) \cdot \mathbf{t}_{\ell} d \gamma \quad \forall \ell \text { edge of } \mathcal{T}_{h}^{\Gamma_{\mathrm{D}}} .
\end{aligned}
$$

Then, a density argument and the fact that $\mathbf{n} \times\left(\left.\mathbf{G}^{\mathrm{I}}\right|_{\Gamma_{\mathrm{D}}} \times \mathbf{n}\right) \in \mathcal{N}_{h}^{2}\left(\Gamma_{\mathrm{D}}\right)$ allow us to conclude that (5.4) holds true for all $\mathbf{G} \in \mathrm{H}^{r}\left(\mathbf{c u r l}, \Omega_{\mathrm{D}}\right)$.

If the data $\mathbf{f}$ of problem $\mathbf{M P}$ is sufficiently smooth, we define

$$
\mathbf{f}_{\mathrm{I}}:=(\mathbf{n} \times \mathbf{f})^{\mathrm{I}_{2}} \times \mathbf{n} ;
$$

that is, $\mathbf{f}_{\mathrm{I}}$ is such that $\mathbf{n} \times \mathbf{f}_{\mathrm{I}}=(\mathbf{n} \times \mathbf{f})^{\mathrm{I}_{2}}$, which means

$$
\mathbf{n} \times \mathbf{f}_{\mathrm{I}} \in \mathcal{N}_{h}^{2}\left(\Gamma_{\mathrm{D}}\right): \quad \int_{\ell} \mathbf{n} \times \mathbf{f}_{\mathrm{I}} \cdot \mathbf{t}_{\ell} d \gamma=\int_{\ell} \mathbf{n} \times \mathbf{f} \cdot \mathbf{t}_{\ell} d \gamma \quad \forall \ell \text { edge of } \mathcal{T}_{h}^{\Gamma_{\mathrm{D}}} .
$$

The following lemma shows that this definition also works under weak smoothness assumptions as those in the previous lemmas:

Lemma 5.3. Let $\mathbf{G} \in \mathrm{H}^{r}\left(\operatorname{curl}, \Omega_{\mathrm{D}}\right)$, with $r>1 / 2$, and let $\mathbf{g}=\left.\mathbf{G}\right|_{\Gamma_{\mathrm{D}}} \times \mathbf{n}$. Then $\mathrm{g}_{\mathrm{I}}:=(\mathbf{n} \times \mathbf{g})^{\mathrm{I}_{2}} \times \mathbf{n}$ is well defined and satisfies

$$
\mathbf{n} \times \mathbf{g}_{\mathrm{I}}=\mathbf{n} \times\left(\left.\mathbf{G}^{\mathrm{I}}\right|_{\Gamma_{\mathrm{D}}} \times \mathbf{n}\right) \quad \text { on } \Gamma_{\mathrm{D}}
$$

Proof. As a consequence of Lemma 5.2, $(\mathbf{n} \times \mathbf{g})^{\mathrm{I}_{2}}=\left[\mathbf{n} \times\left(\left.\mathbf{G}\right|_{\Gamma_{\mathrm{D}}} \times \mathbf{n}\right)\right]^{\mathrm{I}_{2}}$ is well defined. Hence $\mathbf{g}_{\mathrm{I}}:=(\mathbf{n} \times \mathbf{g})^{\mathrm{I}_{2}} \times \mathbf{n}$ is well defined too. Moreover, since according to this lemma (5.4) holds true for $\mathbf{G} \in \mathrm{H}^{r}\left(\mathbf{c u r l}, \Omega_{\mathrm{D}}\right)$, then,

$$
\mathbf{n} \times \mathbf{g}_{\mathrm{I}}=(\mathbf{n} \times \mathbf{g})^{\mathrm{I}_{2}}=\left[\mathbf{n} \times\left(\left.\mathbf{G}\right|_{\Gamma_{\mathrm{D}}} \times \mathbf{n}\right)\right]^{\mathrm{I}_{2}}=\mathbf{n} \times\left(\left.\mathbf{G}^{\mathrm{I}}\right|_{\Gamma_{\mathrm{D}}} \times \mathbf{n}\right) \quad \text { on } \Gamma_{\mathrm{D}}
$$

Now we are in a position to discretize problem MP. We introduce the following finite-dimensional spaces:

$$
\begin{aligned}
& \mathcal{V}_{h}:=\left\{\mathbf{G}_{h} \in \mathcal{N}_{h}(\Omega): \operatorname{curl} \mathbf{G}_{h}=\mathbf{0} \text { in } \Omega_{\mathrm{D}}\right\} \\
& \mathcal{V}_{h}^{0}:=\left\{\mathbf{G}_{h} \in \mathcal{V}_{h}: \mathbf{G}_{h} \times \mathbf{n}=\mathbf{0} \text { on } \Gamma_{\mathrm{D}}\right\}
\end{aligned}
$$

Finally, we define the discrete magnetic problem as follows:

Problem DMP: Find $\mathbf{H}_{h} \in \mathcal{V}_{h}$ such that

$$
\begin{aligned}
& \mathbf{H}_{h} \times \mathbf{n}=\mathbf{f}_{\mathrm{I}} \quad \text { on } \Gamma_{\mathrm{D}} \text {, } \\
& \mathrm{i} \omega \int_{\Omega} \mu \mathbf{H}_{h} \cdot \overline{\mathbf{G}}_{h}+\int_{\Omega_{\mathrm{C}}} \frac{1}{\sigma} \operatorname{curl} \mathbf{H}_{h} \cdot \operatorname{curl} \overline{\mathbf{G}}_{h}=0 \quad \forall \mathbf{G}_{h} \in \mathcal{V}_{h}^{0} .
\end{aligned}
$$

It is straightforward to prove existence and uniqueness of solution for this problem under mild smoothness assumptions on the solution of problem MP. Moreover, an error estimate can be deduced from the standard finite element approximation theory: 
THEOREM 5.4. Let us assume that the solution $\mathbf{H}$ of problem $\mathbf{M P}$ satisfies $\left.\mathbf{H}\right|_{\Omega_{\mathrm{C}}} \in$ $\mathrm{H}^{r}\left(\mathbf{c u r l}, \Omega_{\mathrm{C}}\right)$ and $\left.\mathbf{H}\right|_{\Omega_{\mathrm{D}}} \in \mathrm{H}^{r}\left(\Omega_{\mathrm{D}}\right)^{3}$, with $r \in\left(\frac{1}{2}, 1\right]$. Then, $\mathbf{f}_{\mathrm{I}}$ is well defined by (5.6), problem DMP attains a unique solution $\mathbf{H}_{h}$, and

$$
\left\|\mathbf{H}-\mathbf{H}_{h}\right\|_{\mathrm{H}(\mathbf{c u r l}, \Omega)} \leq C h^{r}\left[\|\mathbf{H}\|_{\mathrm{H}^{r}\left(\mathbf{c u r l}, \Omega_{\mathrm{C}}\right)}+\|\mathbf{H}\|_{\mathrm{H}^{r}\left(\Omega_{\mathrm{D}}\right)^{3}}\right] .
$$

Proof. Since $\mathbf{H} \in \mathcal{V}$, then $\operatorname{curl} \mathbf{H}=\mathbf{0}$ in $\Omega_{\mathrm{D}}$. Hence, $\left.\mathbf{H}\right|_{\Omega_{\mathrm{D}}} \in \mathrm{H}^{r}\left(\operatorname{curl}, \Omega_{\mathrm{D}}\right)$. Therefore, according to Lemma 5.1, its Nédélec interpolant $\mathbf{H}^{\mathrm{I}} \in \mathcal{N}_{h}(\Omega)$ is well defined and satisfies

$$
\left\|\mathbf{H}-\mathbf{H}^{\mathrm{I}}\right\|_{\mathrm{H}(\mathbf{c u r l}, \Omega)} \leq C h^{r}\left[\|\mathbf{H}\|_{\mathrm{H}^{r}\left(\mathbf{c u r l}, \Omega_{\mathrm{C}}\right)}+\|\mathbf{H}\|_{\mathrm{H}^{r}\left(\Omega_{\mathrm{D}}\right)^{3}}\right] .
$$

Moreover, the arguments of Remark 5.6 in [19] can be extended to this case to prove that curl $\left.\mathbf{H}\right|_{\Omega_{\mathrm{D}}}=\mathbf{0}$ implies curl $\left.\mathbf{H}^{\mathrm{I}}\right|_{\Omega_{\mathrm{D}}}=\mathbf{0}$. Therefore, $\mathbf{H}^{\mathrm{I}} \in \mathcal{V}_{h}$.

On the other hand, because of Lemma 5.3, $\mathrm{f}_{\mathrm{I}}$ is well defined by (5.6) and satisfies $\mathbf{f}_{\mathrm{I}}=\left.\mathbf{H}^{\mathrm{I}}\right|_{\Gamma_{\mathrm{D}}} \times \mathbf{n}$ on $\Gamma_{\mathrm{D}}$. Thus, we have proved that there exists $\mathbf{H}^{\mathrm{I}} \in \mathcal{V}_{h}$ such that $\mathbf{H}^{\mathrm{I}} \times \mathbf{n}=\mathbf{f}_{\mathrm{I}}$ on $\Gamma_{\mathrm{D}}$. Hence, since $\mathcal{V}_{h}^{0} \subset \mathcal{V}^{0}$, the arguments in the proof of Theorem 3.1 also apply to problem DMP allowing us to prove existence and uniqueness of a solution $\mathbf{H}_{h}$ of this problem.

Finally, to prove the error estimate, notice that since $\mathcal{V}_{h}^{0} \subset \mathcal{V}^{0}$, then

$$
a\left(\mathbf{H}-\mathbf{H}_{h}, \mathbf{G}_{h}\right)=0 \quad \forall \mathbf{G}_{h} \in \mathcal{V}_{h}^{0} .
$$

Hence, since $\mathbf{H}_{h} \times \mathbf{n}=\mathbf{f}_{\mathrm{I}}=\mathbf{H}^{\mathrm{I}} \times \mathbf{n}$ on $\Gamma_{\mathrm{D}}$, then $\mathbf{H}_{h}-\mathbf{H}^{\mathrm{I}} \in \mathcal{V}_{h}^{0}$. Therefore, because of this and (3.4),

$$
\begin{aligned}
\alpha\left\|\mathbf{H}-\mathbf{H}_{h}\right\|_{\mathrm{H}(\mathbf{c u r l}, \Omega)}^{2} & \leq\left|a\left(\mathbf{H}-\mathbf{H}_{h}, \mathbf{H}-\mathbf{H}_{h}\right)\right|=\left|a\left(\mathbf{H}-\mathbf{H}_{h}, \mathbf{H}-\mathbf{H}^{\mathrm{I}}\right)\right| \\
& \leq C\left\|\mathbf{H}-\mathbf{H}_{h}\right\|_{\mathrm{H}(\mathbf{c u r l}, \Omega)}\left\|\mathbf{H}-\mathbf{H}^{\mathrm{I}}\right\|_{\mathrm{H}(\mathbf{c u r l}, \Omega)},
\end{aligned}
$$

which together with estimate (5.7) allow us to conclude the proof. $\square$

5.2. Discretizing the magnetic potential. Problem DMP is actually just a "theoretical" method in that its solution requires to impose somehow the curl-free condition in the definition of $\mathcal{V}_{h}$ to trial and test functions. In what follows we show how to deal efficiently with this curl-free condition by introducing a discrete multivalued magnetic potential in the dielectric domain.

We assume that the cut surfaces $\Sigma_{j}$ are polyhedral and that the meshes are compatible with them, in the sense that each $\bar{\Sigma}_{j}$ is union of faces of tetrahedra $K \in \mathcal{T}_{h}$, for each mesh $\mathcal{T}_{h}$. Therefore, $\mathcal{T}_{h}^{\Omega_{\mathrm{D}}}:=\left\{K \in \mathcal{T}_{h}: K \subset \bar{\Omega}_{\mathrm{D}}\right\}$ can also be seen as a mesh of $\widetilde{\Omega}_{\mathrm{D}}$.

Firstly, we introduce an approximation of the space $\Theta$. Let us denote

$$
\mathcal{L}_{h}\left(\widetilde{\Omega}_{\mathrm{D}}\right):=\left\{\widetilde{\Psi}_{h} \in \mathrm{H}^{1}\left(\widetilde{\Omega}_{\mathrm{D}}\right):\left.\widetilde{\Psi}_{h}\right|_{K} \in \mathcal{P}_{1}(K) \forall K \in \mathcal{T}_{h}^{\Omega_{\mathrm{D}}}\right\} .
$$

Then, we consider the family of finite dimensional subspaces of $\Theta$ given by

$$
\Theta_{h}:=\left\{\widetilde{\Psi}_{h} \in \mathcal{L}_{h}\left(\widetilde{\Omega}_{\mathrm{D}}\right): \llbracket \widetilde{\Psi}_{h} \rrbracket_{\Sigma_{j}}=\text { constant }, j=1, \ldots, J\right\} .
$$

The following lemma shows that the curl-free vector fields in $\mathcal{N}_{h}\left(\Omega_{\mathrm{D}}\right)$ admit a multivalued potential in $\Theta_{h}$ : 
Lemma 5.5. Let $\mathbf{G}_{h} \in \mathrm{L}^{2}\left(\Omega_{\mathrm{D}}\right)^{3}$. Then $\mathbf{G}_{h} \in \mathcal{N}_{h}\left(\Omega_{\mathrm{D}}\right)$ with $\operatorname{curl} \mathbf{G}_{h}=\mathbf{0}$ in $\Omega_{\mathrm{D}}$ if and only if there exists $\widetilde{\Psi}_{h} \in \Theta_{h}$ such that $\mathbf{G}_{h}=\operatorname{grad} \widetilde{\Psi}_{h}$ in $\Omega_{\mathrm{D}}$. Such $\widetilde{\Psi}_{h}$ is unique up to an additive constant.

Proof. According to (4.1), $\operatorname{curl} \mathbf{G}_{h}=\mathbf{0}$ in $\Omega_{\mathrm{D}}$ if and only if there exists $\widetilde{\Psi}_{h} \in \Theta$ such that $\mathbf{G}_{h}=\operatorname{grad} \widetilde{\Psi}_{h}$ in $\widetilde{\Omega}_{\mathrm{D}}$. Moreover, since $\widetilde{\Omega}_{\mathrm{D}}$ is connected, then $\widetilde{\Psi}_{h}$ is unique up to an additive constant. Now, let $K \in \mathcal{T}_{h}^{\Omega_{\mathrm{D}}}$ be a tetrahedron of the mesh. A direct calculation shows that $\mathbf{G}_{h} \in \mathcal{N}(K)$ with $\left.\operatorname{curl} \mathbf{G}_{h}\right|_{K}=\mathbf{0}$ if and only if $\left.\mathbf{G}_{h}\right|_{K} \in$ $\mathcal{P}_{0}(K)^{3}$, or, equivalently, if and only if $\left.\widetilde{\Psi}_{h}\right|_{K} \in \mathcal{P}_{1}(K)^{3}$. Thus the lemma follows from the definition of $\Theta_{h}$.

Let us introduce the following families of finite-dimensional approximations of $\mathcal{W}$ and $\mathcal{W}^{0}$, respectively:

$$
\begin{aligned}
& \mathcal{W}_{h}:=\left\{\left(\mathbf{G}_{h}, \widetilde{\Psi}_{h}\right) \in \mathcal{N}_{h}\left(\Omega_{\mathrm{C}}\right) \times\left(\Theta_{h} / \mathbb{C}\right):\left(\mathbf{G}_{h} \mid \operatorname{grad} \widetilde{\Psi}_{h}\right) \in \mathrm{H}(\operatorname{curl}, \Omega)\right\} \\
& \mathcal{W}_{h}^{0}:=\left\{\left(\mathbf{G}_{h}, \widetilde{\Psi}_{h}\right) \in \mathcal{W}_{h}: \operatorname{grad} \widetilde{\Psi}_{h} \times \mathbf{n}=\mathbf{0} \text { on } \Gamma_{\mathrm{D}}\right\}
\end{aligned}
$$

By virtue of Lemma $5.5, \mathcal{W}_{h}$ and $\mathcal{W}_{h}^{0}$ are isomorphically equivalent to $\mathcal{V}_{h}$ and $\mathcal{V}_{h}^{0}$, respectively. Thus, we define the following discrete problem which turns out to be equivalent to problem DMP:

Problem DHP: To find $\left(\mathbf{H}_{h}, \widetilde{\Phi}_{h}\right) \in \mathcal{W}_{h}$ such that

$$
\begin{aligned}
& \operatorname{grad} \widetilde{\Phi}_{h} \times \mathbf{n}=\mathbf{f}_{\mathrm{I}} \quad \text { on } \Gamma_{\mathrm{D}} \\
& \begin{aligned}
\mathrm{i} \omega \int_{\Omega_{\mathrm{C}}} \mu \mathbf{H}_{h} \cdot \overline{\mathbf{G}}_{h} & +\int_{\Omega_{\mathrm{C}}} \frac{1}{\sigma} \operatorname{curl} \mathbf{H}_{h} \cdot \operatorname{curl} \overline{\mathbf{G}}_{h} \\
& +\mathrm{i} \omega \int_{\Omega_{\mathrm{D}}} \mu \operatorname{grad} \widetilde{\Phi}_{h} \cdot \operatorname{grad} \overline{\widetilde{\Psi}}_{h}=0 \quad \forall\left(\mathbf{G}_{h}, \widetilde{\Psi}_{h}\right) \in \mathcal{W}_{h}^{0}
\end{aligned}
\end{aligned}
$$

Clearly, the following discrete analogue of Lemma 4.1 holds true:

LEMMA 5.6. The pair $\left(\mathbf{H}_{h}, \widetilde{\Phi}_{h}\right)$ is solution of problem DHP if and only if $\left(\mathbf{H}_{h} \mid \operatorname{grad} \widetilde{\Phi}_{h}\right)$ is solution of problem DMP.

As an immediate consequence of these two lemmas, Theorem 5.4 yields an error estimate for the approximation obtained from problem DHP:

Corollary 5.7. Let us assume that the solution $(\mathbf{H}, \widetilde{\Phi})$ of problem $\mathbf{H P}$ satisfies $\mathbf{H} \in \mathrm{H}^{r}\left(\mathbf{c u r l}, \Omega_{\mathrm{C}}\right)$ and $\mathbf{g r a d} \widetilde{\Phi} \in \mathrm{H}^{r}\left(\Omega_{\mathrm{D}}\right)^{3}$, with $r \in\left(\frac{1}{2}, 1\right]$. Then, problem DHP is well posed, it attains a unique solution $\left(\mathbf{H}_{h}, \widetilde{\Phi}_{h}\right)$, and

$$
\begin{aligned}
\left\|\mathbf{H}-\mathbf{H}_{h}\right\|_{\mathrm{H}\left(\mathbf{c u r l}, \Omega_{\mathrm{C}}\right)}+\| \operatorname{grad} \widetilde{\Phi} & -\operatorname{grad} \widetilde{\Phi}_{h} \|_{\mathrm{L}^{2}\left(\Omega_{\mathrm{D}}\right)^{3}} \\
& \leq C h^{r}\left[\|\mathbf{H}\|_{\mathrm{H}^{r}\left(\operatorname{curl}, \Omega_{\mathrm{C}}\right)}+\|\operatorname{grad} \widetilde{\Phi}\|_{\mathrm{H}^{r}\left(\Omega_{\mathrm{D}}\right)^{3}}\right] .
\end{aligned}
$$

6. Computer implementation. For problem DHP to be useful for computational purposes, we have to introduce effective procedures to impose the following constraints:

1. $\left(\mathbf{G}_{h} \mid \operatorname{grad} \widetilde{\Psi}_{h}\right) \in \mathrm{H}(\mathbf{c u r l}, \Omega)$, which arise in the definition of $\mathcal{W}_{h}$;

2. $\llbracket \widetilde{\Psi}_{h} \rrbracket_{\Sigma_{j}}=$ constant, which arise in the definition of $\Theta_{h}$;

3. the boundary condition $\mathbf{g r a d} \widetilde{\Phi}_{h} \times \mathbf{n}=\mathbf{f}_{\mathrm{I}}$ on $\Gamma_{\mathrm{D}}$. 
We fix some notation to deal with these constraints. We choose an orientation for each edge $\ell$ of the mesh $\mathcal{T}_{h}$ and denote $P_{\ell}^{-}$and $P_{\ell}^{+}$its initial and end points, respectively, and $\mathbf{t}_{\ell}$ its unit tangent vector pointing from $P_{\ell}^{-}$to $P_{\ell}^{+}$.

Regarding the first constraint we have the following result:

LEMma 6.1. Let $\left(\mathbf{G}_{h}, \widetilde{\Psi}_{h}\right) \in \mathcal{N}_{h}\left(\Omega_{\mathrm{C}}\right) \times\left(\Theta_{h} / \mathbb{C}\right)$. Then, $\left(\mathbf{G}_{h}, \mid \mathbf{g r a d} \widetilde{\Psi}_{h}\right) \in$ $\mathrm{H}(\mathbf{c u r l}, \Omega)$ if and only if

$$
\int_{\ell} \mathbf{G}_{h} \cdot \mathbf{t}_{\ell} d \gamma=\widetilde{\Psi}_{h}\left(P_{\ell}^{+}\right)-\widetilde{\Psi}_{h}\left(P_{\ell}^{-}\right) \quad \forall \ell \text { edge of } \mathcal{T}_{h}: \ell \subset \Gamma_{\Gamma} .
$$

Proof. Since $\mathbf{G}_{h} \in \mathcal{N}_{h}\left(\Omega_{\mathrm{C}}\right)$ and $\operatorname{grad} \widetilde{\Psi}_{h} \in \mathcal{N}_{h}\left(\Omega_{\mathrm{D}}\right)$, then, $\left(\mathbf{G}_{h} \mid \operatorname{grad} \widetilde{\Psi}_{h}\right) \in$ $\mathrm{H}(\mathbf{c u r l}, \Omega)$ if and only if the tangential traces on $\Gamma_{\mathrm{I}}$ of $\mathbf{G}_{h}$ and $\operatorname{grad} \widetilde{\Psi}_{h}$ coincide; that is, if and only if

$$
\mathbf{n} \times\left(\mathbf{G}_{h} \times \mathbf{n}\right)=\mathbf{n} \times\left(\operatorname{grad} \widetilde{\Psi}_{h} \times \mathbf{n}\right) \quad \text { on } \Gamma_{\mathrm{I}}
$$

Now, the equation above holds true if and only if the degrees of freedom of $\mathbf{G}_{h}$ and $\operatorname{grad} \widetilde{\Psi}_{h}$ coincide on all the edges $\ell \subset \Gamma_{\mathrm{I}}$, and this reads

$$
\int_{\ell} \mathbf{G}_{h} \cdot \mathbf{t}_{\ell} d \gamma=\int_{\ell} \operatorname{grad} \widetilde{\Psi}_{h} \cdot \mathbf{t}_{\ell} d \gamma=\widetilde{\Psi}_{h}\left(P_{\ell}^{+}\right)-\widetilde{\Psi}_{h}\left(P_{\ell}^{-}\right) .
$$

This lemma shows that the constraint $\left(\mathbf{G}_{h} \mid \operatorname{grad} \widetilde{\Psi}_{h}\right) \in \mathrm{H}(\mathbf{c u r l}, \Omega)$ can be readily imposed by eliminating the degrees of freedom of $\mathbf{G}_{h}$ associated with the edges $\ell \subset \Gamma_{\mathrm{I}}$, in terms of those of $\widetilde{\Phi}_{h}$ corresponding to the vertices of the mesh on this interface.

Regarding the second constraint, for $\widetilde{\Psi}_{h} \in \Theta_{h}$, let us denote

$$
c_{h j}:=\llbracket \widetilde{\Psi}_{h} \rrbracket_{\Sigma_{j}}, \quad j=1, \ldots, J .
$$

In order to handle the multivalued character of the functions $\widetilde{\Psi}_{h} \in \Theta_{h}$, for each cut surface $\Sigma_{j}$, we in principle distinguish the degrees of freedom of $\widetilde{\Psi}_{h}$ on $\bar{\Sigma}_{j}^{+}$from those on $\bar{\Sigma}_{j}^{-}$. Then, the latter can be eliminated by using

$$
\left.\widetilde{\Psi}_{h}\right|_{\Sigma_{j}^{-}}=\left.\widetilde{\Psi}_{h}\right|_{\Sigma_{j}^{+}}+\llbracket \widetilde{\Psi}_{h} \rrbracket_{\bar{\Sigma}_{j}}=\left.\widetilde{\Psi}_{h}\right|_{\Sigma_{j}^{+}}+c_{h j}, \quad j=1, \ldots, J
$$

This elimination must be carried out for the solution $\left(\mathbf{H}_{h}, \widetilde{\Phi}_{h}\right) \in \mathcal{W}_{h}$ of problem DHP as well as for the test functions $\left(\mathbf{G}_{h}, \widetilde{\Psi}_{h}\right) \in \mathcal{W}_{h}^{0}$. For the former, the arguments in Remark 4.1 can be repeated at discrete level to show that each jump $\llbracket \widetilde{\Phi}_{h} \rrbracket_{\Sigma_{j}}$ represents the current intensity through the conductor $\Omega_{\mathrm{C}}^{j}$ corresponding to the discrete solution $\left(\mathbf{H}_{h}, \widetilde{\Phi}_{h}\right)$. Because of this, we denote these jumps

$$
I_{h j}:=\llbracket \widetilde{\Phi}_{h} \rrbracket_{\Sigma_{j}}, \quad j=1, \ldots, J .
$$

For $j=1, \ldots, K$ (i.e., for inner conductors $\Omega_{\mathrm{C}}^{j}$ ), $I_{h j}$ are additional unknowns of the discrete problem. Instead, for $j=K+1, \ldots, J$ (i.e., for conductors $\Omega_{\mathrm{C}}^{j}$ going through $\partial \Omega$ ), $I_{h j}$ can be computed in advance from the data of the discrete problem. Indeed, since $\gamma_{j} \subset \Gamma_{\mathrm{D}}$ and $\operatorname{grad} \widetilde{\Phi}_{h} \times \mathbf{n}=\mathbf{f}_{\mathrm{I}}$ on $\Gamma_{\mathrm{D}}$, then,

$$
I_{h j}=\int_{\gamma_{j}} \operatorname{grad} \widetilde{\Phi}_{h} \cdot \mathbf{t}_{j} d \gamma=\int_{\gamma_{j}} \mathbf{n} \times\left(\operatorname{grad} \widetilde{\Phi}_{h} \times \mathbf{n}\right) \cdot \mathbf{t}_{j} d \gamma=\int_{\gamma_{j}} \mathbf{n} \times \mathbf{f}_{\mathrm{I}} \cdot \mathbf{t}_{j} d \gamma
$$


For the test functions $\left(\mathbf{G}_{h}, \widetilde{\Psi}_{h}\right) \in \mathcal{W}_{h}^{0}$, by repeating these arguments and using that $\operatorname{grad} \widetilde{\Psi}_{h} \times \mathbf{n}=\mathbf{0}$ on $\Gamma_{\mathrm{D}}$, we have

$$
c_{h j}=\int_{\gamma_{j}} \mathbf{n} \times\left(\mathbf{g r a d} \widetilde{\Psi}_{h} \times \mathbf{n}\right) \cdot \mathbf{t}_{j} d \gamma=0, \quad j=K+1, \ldots, J
$$

Hence, only the constants $c_{h j}$, for $j=1, \ldots, K$, must be taken into account as genuine degrees of freedom in the definition of $\mathcal{W}_{h}^{0}$.

Remark 6.1. The computed and exact intensities through the conductors $\Omega_{\mathrm{C}}^{j}$, $j=K+1, \ldots, J$, coincide. Indeed, because of (6.1), Lemma 5.3, (5.1), the fact that each $\gamma_{j}$ is union of edges $\ell$ in $\mathcal{T}_{h}$, and Remark 4.1, we have

$$
\begin{aligned}
I_{h j} & =\int_{\gamma_{j}} \mathbf{n} \times \mathbf{f}_{\mathrm{I}} \cdot \mathbf{t}_{j} d \gamma=\int_{\gamma_{j}} \mathbf{n} \times\left[\left.(\operatorname{grad} \widetilde{\Phi})^{\mathrm{I}}\right|_{\Gamma_{\mathrm{D}}} \times \mathbf{n}\right] \cdot \mathbf{t}_{j} d \gamma \\
& =\int_{\gamma_{j}}(\operatorname{grad} \widetilde{\Phi})^{\mathrm{I}} \cdot \mathbf{t}_{j} d \gamma=\int_{\gamma_{j}} \operatorname{grad} \widetilde{\Phi} \cdot \mathbf{t}_{j} d \gamma=\llbracket \widetilde{\Phi} \rrbracket_{\Sigma_{j}}=I_{j} .
\end{aligned}
$$

Regarding the third constraint, we impose the boundary condition by means of a Lagrange multiplier. Let $\widetilde{\Gamma}_{\mathrm{D}}$ be the pseudo-Lipschitz connected polyhedral surface defined by

$$
\widetilde{\Gamma}_{\mathrm{D}}:=\Gamma_{\mathrm{D}} \backslash \bigcup_{j=K+1}^{J}\left(\bar{\Sigma}_{j} \cap \Gamma_{\mathrm{D}}\right) .
$$

Let

$$
\begin{aligned}
& \mathcal{L}_{h}\left(\widetilde{\Gamma}_{\mathrm{D}}\right):=\left\{\nu_{h} \in \mathrm{H}^{1}\left(\widetilde{\Gamma}_{\mathrm{D}}\right):\left.\nu_{h}\right|_{T} \in \mathcal{P}_{1}(T) \forall T \in \mathcal{T}_{h}^{\Gamma_{\mathrm{D}}}\right\}, \\
& \mathcal{L}_{h}\left(\Gamma_{\mathrm{D}}\right):=\left\{\nu_{h} \in \mathrm{H}^{1}\left(\Gamma_{\mathrm{D}}\right):\left.\nu_{h}\right|_{T} \in \mathcal{P}_{1}(T) \forall T \in \mathcal{T}_{h}^{\Gamma_{\mathrm{D}}}\right\} .
\end{aligned}
$$

Hence, given $\nu_{h} \in \mathcal{L}_{h}\left(\widetilde{\Gamma}_{\mathrm{D}}\right)$, we have that $\nu_{h} \in \mathcal{L}_{h}\left(\Gamma_{\mathrm{D}}\right)$ if and only if $\llbracket \nu_{h} \rrbracket_{\bar{\Sigma}_{j} \cap \Gamma_{\mathrm{D}}}=0$ for $j=K+1, \ldots, J$.

Let $\mathbf{g r a d}{ }_{\Gamma}$ denote the surface gradient operator. Since we will use this operator acting only on piecewise linear functions, we give a definition valid in this case (for its general definition on polyhedral surfaces, see $[14,15])$ :

$$
\operatorname{grad}_{\Gamma}: \mathcal{L}_{h}\left(\widetilde{\Gamma}_{\mathrm{D}}\right) \longrightarrow \mathrm{L}_{\mathbf{t}}^{2}\left(\Gamma_{\mathrm{D}}\right)^{3}:=\left\{\boldsymbol{\varphi} \in \mathrm{L}^{2}\left(\Gamma_{\mathrm{D}}\right)^{3}: \boldsymbol{\varphi} \cdot \mathbf{n}=0 \text { on } \Gamma_{\mathrm{D}}\right\}
$$

is defined, on each element $T \in \mathcal{T}_{h}^{\Gamma_{\mathrm{D}}}$, by $\left.\left(\operatorname{grad}_{\Gamma} \nu_{h}\right)\right|_{T}=\nabla_{2}\left(\left.\nu_{h}\right|_{T}\right)$, where $\nabla_{2}$ is the usual gradient of a function of two variables; i.e., using local coordinates $(\xi, \eta, \zeta)$ such that $T$ is in the plane $\zeta=0$,

$$
\left.\left(\operatorname{grad}_{\Gamma} \nu_{h}\right)\right|_{T}:=\left(\frac{\partial\left(\left.\nu_{h}\right|_{T}\right)}{\partial \xi}, \frac{\partial\left(\left.\nu_{h}\right|_{T}\right)}{\partial \eta}, 0\right)
$$

For all $\widetilde{\Psi}_{h} \in \mathcal{L}_{h}\left(\widetilde{\Omega}_{\mathrm{D}}\right)$, we have $\left.\widetilde{\Psi}_{h}\right|_{\Gamma_{\mathrm{D}}} \in \mathcal{L}_{h}\left(\widetilde{\Gamma}_{\mathrm{D}}\right)$, and it is straightforward to show that

$$
\operatorname{grad} \widetilde{\operatorname{rad}}_{\Gamma}\left(\left.\widetilde{\Psi}_{h}\right|_{\widetilde{\Gamma}_{\mathrm{D}}}\right)=\mathbf{n} \times\left[\left.\left(\operatorname{grad} \widetilde{\Psi}_{h}\right)\right|_{\Gamma_{\mathrm{D}}} \times \mathbf{n}\right] \quad \text { a.e. in } \Gamma_{\mathrm{D}}
$$


The following lemma provides a weak formulation of the boundary condition (5.8) in problem DHP:

Lemma 6.2. Let $\widetilde{\Psi} \in \Theta$ be such that $\operatorname{grad} \widetilde{\Psi} \in \mathrm{H}^{r}\left(\Omega_{\mathrm{D}}\right)$ with $r>1 / 2$. Let $\mathbf{g}=\left.\operatorname{grad} \widetilde{\Psi}\right|_{\Gamma_{\mathrm{D}}} \times \mathbf{n}$ and $\mathbf{g}_{\mathrm{I}}=(\mathbf{n} \times \mathbf{g})^{\mathrm{I}_{2}} \times \mathbf{n}$ (well defined because of Lemma 5.3). Let $\widetilde{\Psi}_{h} \in \Theta_{h}$ be such that

$$
\llbracket \widetilde{\Psi}_{h} \rrbracket_{\Sigma_{j}}=\int_{\gamma_{j}} \mathbf{n} \times \mathbf{g}_{\mathrm{I}} \cdot \mathbf{t}_{j} d \gamma, \quad j=K+1, \ldots, J .
$$

Then, $\operatorname{grad} \widetilde{\Psi}_{h} \times \mathbf{n}=\mathbf{g}_{\mathrm{I}}$ on $\Gamma_{\mathrm{D}}$ if and only if

$$
\int_{\Gamma_{\mathrm{D}}} \operatorname{grad}_{\Gamma} \widetilde{\Psi}_{h} \cdot \operatorname{grad}_{\Gamma} \bar{\nu}_{h} d \Gamma=\int_{\Gamma_{\mathrm{D}}} \mathbf{n} \times \mathbf{g}_{\mathrm{I}} \cdot \operatorname{grad}_{\Gamma} \bar{\nu}_{h} d \Gamma \quad \forall \nu_{h} \in \mathcal{L}_{h}\left(\Gamma_{\mathrm{D}}\right) / \mathbb{C}
$$

Proof. If grad $\widetilde{\Psi}_{h} \times \mathbf{n}=\mathrm{g}_{\mathrm{I}}$ on $\Gamma_{\mathrm{D}}$, then, because of (6.3), we have (6.5).

Conversely, let us assume that (6.5) holds true. Since $\widetilde{\Psi} \in \mathrm{H}^{1+r}\left(\widetilde{\Omega}_{\mathrm{D}}\right) / \mathbb{C}$ with $r>1 / 2$, then its Lagrange interpolant $\widetilde{\Psi}^{\mathrm{L}} \in \mathcal{L}_{h}\left(\widetilde{\Omega}_{\mathrm{D}}\right) / \mathbb{C}$ is well defined. Given a cut surface $\Sigma_{j}, j=1, \cdots, J$, for all the vertices $P$ of $\mathcal{T}_{h}$ such that $P \in \Sigma_{j}$, we have $\llbracket \widetilde{\Psi}^{\mathrm{L}}(P) \rrbracket_{\Sigma_{j}}=\llbracket \widetilde{\Psi}(P) \rrbracket_{\Sigma_{j}}=$ constant (the same for all such $P$ ). Then $\llbracket \widetilde{\Psi}^{\mathrm{L}} \rrbracket_{\Sigma_{j}}=$ constant, and hence $\widetilde{\Psi}^{\mathrm{L}} \in \Theta_{h} / \mathbb{C}$. Thus, because of Lemma 5.5, grad $\widetilde{\Psi}^{\mathrm{L}} \in \mathcal{N}_{h}\left(\Omega_{\mathrm{D}}\right)$.

On the other hand, let $(\operatorname{grad} \widetilde{\Psi})^{\mathrm{I}} \in \mathcal{N}_{h}\left(\Omega_{\mathrm{D}}\right)$ be the Nédélec interpolant of $\operatorname{grad} \widetilde{\Psi}$. We have $\int_{\ell}(\operatorname{grad} \widetilde{\Psi})^{\mathrm{I}} \cdot \mathbf{t}_{\ell} d \gamma=\int_{\ell} \operatorname{grad} \widetilde{\Psi} \widetilde{\mathrm{T}}^{\mathrm{L}} \cdot \mathbf{t}_{\ell} d \gamma \quad \forall \ell$ edge of $\mathcal{T}_{h}^{\Omega_{\mathrm{D}}}$. Indeed, if grad $\widetilde{\Psi}$ were smooth (v.g., grad $\left.\widetilde{\Psi} \in \mathrm{H}^{2}\left(\Omega_{\mathrm{D}}\right)^{3}\right)$, then

$$
\begin{aligned}
\int_{\ell}(\operatorname{grad} \widetilde{\Psi})^{\mathrm{I}} \cdot \mathbf{t}_{\ell} d \gamma & =\int_{\ell} \operatorname{grad} \widetilde{\Psi} \cdot \mathbf{t}_{\ell} d \gamma=\widetilde{\Psi}\left(P_{\ell}^{+}\right)-\widetilde{\Psi}\left(P_{\ell}^{-}\right) \\
& =\widetilde{\Psi}^{\mathrm{L}}\left(P_{\ell}^{+}\right)-\widetilde{\Psi}^{\mathrm{L}}\left(P_{\ell}^{-}\right)=\int_{\ell} \operatorname{grad} \widetilde{\Psi}^{\mathrm{L}} \cdot \mathbf{t}_{\ell} d \gamma .
\end{aligned}
$$

Hence, because of a density argument, this is also true for $\operatorname{grad} \widetilde{\Psi} \in \mathrm{H}^{r}\left(\operatorname{curl}, \Omega_{\mathrm{D}}\right)^{3}$.

Therefore, since we have shown that $\operatorname{grad} \widetilde{\Psi}^{\mathrm{L}} \in \mathcal{N}_{h}\left(\Omega_{\mathrm{D}}\right)$ and that its degrees of freedom coincide with those of $(\operatorname{grad} \widetilde{\Psi})^{\mathrm{I}}$ for all edges $\ell$ of $\mathcal{T}_{h}^{\Omega_{\mathrm{D}}}$, then, we have

$$
(\operatorname{grad} \widetilde{\Psi})^{\mathrm{I}}=\operatorname{grad} \widetilde{\Psi}^{L} \quad \text { in } \bar{\Omega}_{D} .
$$

Consequently,

$$
\begin{aligned}
\llbracket \widetilde{\Psi}^{\mathrm{L}} \rrbracket_{\Sigma_{j}} & =\int_{\gamma_{j}} \operatorname{grad} \widetilde{\Psi}^{\mathrm{L}} \cdot \mathbf{t}_{j} d \gamma=\int_{\gamma_{j}}(\operatorname{grad} \widetilde{\Psi})^{\mathrm{I}} \cdot \mathbf{t}_{j} d \gamma \\
& =\int_{\gamma_{j}} \mathbf{n} \times\left[\left.(\operatorname{grad} \widetilde{\Psi})^{\mathrm{I}}\right|_{\Gamma_{\mathrm{D}}} \times \mathbf{n}\right] \cdot \mathbf{t}_{j} d \gamma=\int_{\gamma_{j}} \mathbf{n} \times \mathbf{g}_{\mathrm{I}} \cdot \mathbf{t}_{j} d \gamma, \quad j=K+1, \cdots, J,
\end{aligned}
$$

the last equality because of Lemma 5.3.

Let $\nu_{h}:=\left.\left(\widetilde{\Psi}_{h}-\widetilde{\Psi}^{\mathrm{L}}\right)\right|_{\widetilde{\Gamma}_{\mathrm{D}}} \in \mathcal{L}_{h}\left(\widetilde{\Gamma}_{\mathrm{D}}\right) / \mathbb{C}$. Because of the equation above and (6.4), we have

$$
\llbracket \nu_{h} \rrbracket_{\bar{\Sigma}_{j} \cap \Gamma_{\mathrm{D}}}=\llbracket \widetilde{\Psi}_{h} \rrbracket_{\Sigma_{j}}-\llbracket \widetilde{\Psi}^{\mathrm{L}} \rrbracket_{\Sigma_{j}}=0, \quad j=K+1, \cdots, J .
$$


Then, $\nu_{h} \in \mathcal{L}_{h}\left(\Gamma_{\mathrm{D}}\right) / \mathbb{C}$, and, because of (6.3), (6.6), and Lemma 5.3, we have

$$
\operatorname{grad}_{\Gamma} \nu_{h}=\operatorname{grad}_{\Gamma}\left(\left.\widetilde{\Psi}_{h}\right|_{\widetilde{\Gamma}_{\mathrm{D}}}\right)-\mathbf{n} \times\left[\left.\left(\operatorname{grad} \widetilde{\Psi}^{\mathrm{L}}\right)\right|_{\Gamma_{\mathrm{D}}} \times \mathbf{n}\right]=\operatorname{grad}_{\Gamma}\left(\left.\widetilde{\Psi}_{h}\right|_{\widetilde{\Gamma}_{\mathrm{D}}}\right)-\mathbf{n} \times \mathbf{g}_{\mathrm{I}}
$$

Thus, using this $\nu_{h}$ in (6.5), we obtain

$$
\operatorname{grad}_{\Gamma}\left(\widetilde{\Psi}_{h} \widetilde{\Gamma}_{\Gamma_{\mathrm{D}}}\right)-\mathbf{n} \times \mathbf{g}_{\mathrm{I}}=\mathbf{0} .
$$

Hence, by using again (6.3), we conclude the proof.

Now we are in a position to set a new discrete problem including the three constraints as we have just described. To this aim, we introduce the following discrete spaces:

$$
\begin{aligned}
\mathcal{Z}_{h}:=\left\{\left(\mathbf{G}_{h}, \widetilde{\Psi}_{h}, c_{h}\right) \in \mathcal{N}_{h}\left(\Omega_{\mathrm{C}}\right) \times\left(\Theta_{h} / \mathbb{C}\right) \times \mathbb{C}^{J}: \llbracket \widetilde{\Psi}_{h} \rrbracket_{\Sigma_{j}}=c_{h j}, j=1, \ldots, J\right. \\
\text { and } \left.\int_{\ell} \mathbf{G}_{h} \cdot \mathbf{t}_{\ell} d \gamma=\widetilde{\Psi}_{h}\left(P_{\ell}^{+}\right)-\widetilde{\Psi}_{h}\left(P_{\ell}^{-}\right) \forall \ell \text { edge } \mathcal{T}_{h}: \ell \subset \Gamma_{\mathrm{I}}\right\}, \\
\mathcal{Z}_{h}^{0}:=\left\{\left(\mathbf{G}_{h}, \widetilde{\Psi}_{h}, c_{h}\right) \in \mathcal{Z}_{h}: c_{h j}=0, j=K+1, \ldots, J\right\} .
\end{aligned}
$$

The new discrete problem, which will be shown to be equivalent to problem DHP in the next theorem, is the following one:

Problem DLP: To find $\left(\mathbf{H}_{h}, \widetilde{\Phi}_{h}, I_{h}\right) \in \mathcal{Z}_{h}$ and $\lambda_{h} \in \mathcal{L}_{h}\left(\Gamma_{\mathrm{D}}\right) / \mathbb{C}$, such that

$$
\begin{aligned}
& I_{h j}=\int_{\gamma_{j}}\left(\mathbf{n} \times \mathbf{f}_{\mathrm{I}}\right) \cdot \mathbf{t}_{j} d \gamma, \quad j=K+1, \ldots, J \\
& \mathrm{i} \omega \int_{\Omega_{\mathrm{C}}} \mu \mathbf{H}_{h} \cdot \overline{\mathbf{G}}_{h}+\int_{\Omega_{\mathrm{C}}} \frac{1}{\sigma} \operatorname{curl} \mathbf{H}_{h} \cdot \operatorname{curl} \overline{\mathbf{G}}_{h}+\mathrm{i} \omega \int_{\Omega_{\mathrm{D}}} \mu \operatorname{grad} \widetilde{\Phi}_{h} \cdot \operatorname{grad} \overline{\widetilde{\Psi}}_{h} \\
& +\int_{\Gamma_{\mathrm{D}}} \operatorname{grad}_{\Gamma} \lambda_{h} \cdot \operatorname{grad} \widetilde{\operatorname{rad}}_{\Gamma} \overline{\widetilde{\Psi}}_{h} d \Gamma=0 \quad \forall\left(\mathbf{G}_{h}, \widetilde{\Psi}_{h}, c_{h}\right) \in \mathcal{Z}_{h}^{0}, \\
& \int_{\Gamma_{\mathrm{D}}} \operatorname{grad}_{\Gamma} \widetilde{\Phi}_{h} \cdot \operatorname{grad}_{\Gamma} \bar{\nu}_{h} d \Gamma=\int_{\Gamma_{\mathrm{D}}}\left(\mathbf{n} \times \mathbf{f}_{\mathrm{I}}\right) \cdot \operatorname{grad}_{\Gamma} \bar{\nu}_{h} d \Gamma \quad \forall \nu_{h} \in \mathcal{L}_{h}\left(\Gamma_{\mathrm{D}}\right) / \mathbb{C}
\end{aligned}
$$

First we prove that problem DLP is well posed:

THEOREM 6.3. Let $\mathbf{f}_{\mathrm{I}}$ be any vector field defined on $\Gamma_{\mathrm{D}}$, such that the integrals in the right hand sides of equations (6.7) and (6.9) are well defined. Then, problem DLP attains a unique solution.

Proof. Problem DLP reduces to a linear system with the same number of equations than unknowns. Then, it is enough to prove that, for $\mathbf{f}_{I}=\mathbf{0}$, this problem attains only the null solution. So let $\left(\mathbf{H}_{h}, \widetilde{\Phi}_{h}, I_{h}\right) \in \mathcal{Z}_{h}$ and $\lambda_{h} \in \mathcal{L}_{h}\left(\Gamma_{\mathrm{D}}\right) / \mathbb{C}$ satisfying (6.7)-(6.9) with $\mathbf{f}_{\mathrm{I}}=\mathbf{0}$.

Equation (6.7) implies $I_{h j}=0$, for $j=K+1, \cdots, J$; hence, $\left(\mathbf{H}_{h}, \widetilde{\Phi}_{h}, I_{h}\right) \in \mathcal{Z}_{h}^{0}$ and $\llbracket \widetilde{\Phi}_{h} \rrbracket_{\Sigma_{j}}=I_{h j}=0$ too. Thus, if we define $\nu_{h}:=\left.\widetilde{\Phi}_{h}\right|_{\widetilde{\Gamma}_{\mathrm{D}}} \in \mathcal{L}_{h}\left(\widetilde{\Gamma}_{\mathrm{D}}\right) / \mathbb{C}$, we have $\llbracket \nu_{h} \rrbracket_{\bar{\Sigma}_{j} \cap \Gamma_{\mathrm{D}}}=0$, for $j=K+1, \ldots, J$, and, then, $\nu_{h} \in \mathcal{L}_{h}\left(\Gamma_{\mathrm{D}}\right) / \mathbb{C}$.

Now, by testing $(6.9)$ with this $\nu_{h}$, we obtain $\operatorname{grad}_{\Gamma}\left(\left.\widetilde{\Phi}_{h}\right|_{\Gamma_{\mathrm{D}}}\right)=\mathbf{0}$. Hence, by testing (6.8) with $\left(\mathbf{H}_{h}, \widetilde{\Phi}_{h}, I_{h}\right)$ (which was already shown to belong to $\mathcal{Z}_{h}^{0}$ ), we obtain

$$
\mathrm{i} \omega \int_{\Omega_{\mathrm{C}}} \mu\left|\mathbf{H}_{h}\right|^{2}+\int_{\Omega_{\mathrm{C}}} \frac{1}{\sigma}\left|\operatorname{curl} \mathbf{H}_{h}\right|^{2}+\mathrm{i} \omega \int_{\Omega_{\mathrm{D}}} \mu\left|\operatorname{grad} \widetilde{\Phi}_{h}\right|^{2}=0 .
$$


Hence, $\mathbf{H}_{h}=\mathbf{0}$ in $\Omega_{\mathrm{C}}$ and $\operatorname{grad} \widetilde{\Phi}_{h}=\mathbf{0}$ in $\Omega_{\mathrm{D}}$. Consequently, $\widetilde{\Phi}_{h}=0$ in $\mathcal{L}_{h}\left(\widetilde{\Omega}_{\mathrm{D}}\right) / \mathbb{C}$ and $I_{h j}=\llbracket \widetilde{\Phi}_{h} \rrbracket_{\Sigma_{j}}=0$ for $j=1, \cdots, K$ too.

Thus, it only remains to prove that $\lambda_{h}=0$. To do this, let us show first that there exists $\left(\mathbf{G}_{h}, \widetilde{\Psi}_{h}, c_{h}\right) \in \mathcal{Z}_{h}^{0}$ satisfying $\left.\widetilde{\Psi}_{h}\right|_{\Gamma_{\mathrm{D}}}=\lambda_{h}$. Indeed, let $\widetilde{\Psi}_{h} \in \mathcal{L}_{h}\left(\widetilde{\Omega}_{\mathrm{D}}\right) / \mathbb{C}$ be the unique function in this space satisfying for each vertex $P$ of $\mathcal{T}_{h}^{\Omega_{\mathrm{D}}}$,

$$
\begin{array}{ll}
\widetilde{\Psi}_{h}(P)=\lambda_{h}(P), & \text { if } P \in \Gamma_{\mathrm{D}}, \\
\widetilde{\Psi}_{h}(P)=0, & \text { if } P \notin \Gamma_{\mathrm{D}} .
\end{array}
$$

Let $c_{h j}=\llbracket \widetilde{\Psi}_{h} \rrbracket_{\Sigma_{j}}, j=1, \cdots, J$. Because of the definition of $\widetilde{\Psi}_{h}$, clearly $c_{h}=0$. Then $\widetilde{\Psi}_{h} \in \Theta_{h}$. Finally, let $\mathbf{G}_{h} \in \mathcal{N}_{h}\left(\Omega_{\mathrm{C}}\right)$ be the unique function in this space satisfying for each edge $\ell$ of $\mathcal{T}_{h}$ such that $\ell \subset \bar{\Omega}_{\mathrm{C}}$,

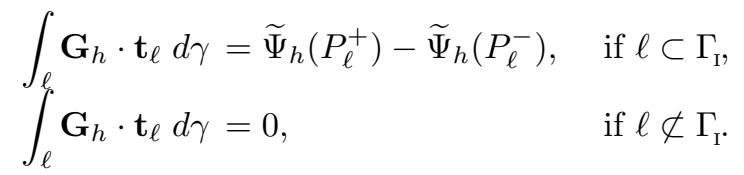

Therefore, $\left(\mathbf{G}_{h}, \widetilde{\Psi}_{h}, c_{h}\right) \in \mathcal{Z}_{h}^{0}$ and $\left.\widetilde{\Psi}_{h}\right|_{\Gamma_{\mathrm{D}}}=\lambda_{h}$. Now, by testing (6.8) with this $\left(\mathbf{G}_{h}, \widetilde{\Psi}_{h}, c_{h}\right)$, since we already know that $\mathbf{H}_{h}=\mathbf{0}$ in $\Omega_{\mathrm{C}}$ and $\operatorname{grad} \widetilde{\Phi}_{h}=0$ in $\Omega_{\mathrm{D}}$, we obtain

$$
\int_{\Gamma_{\mathrm{D}}}\left|\operatorname{grad}_{\Gamma} \lambda_{h}\right|^{2} d \Gamma=0
$$

Then, $\lambda_{h}=0$ in $\mathcal{L}_{h}\left(\Gamma_{\mathrm{D}}\right) / \mathbb{C}$, and we conclude the proof.

Now it is very simple to show that problems DHP and DLP are equivalent:

THEOREM 6.4. Let us assume that the solution $(\mathbf{H}, \widetilde{\Phi})$ of problem $\mathbf{H P}$ satisfies $\mathbf{H} \in \mathrm{H}^{r}\left(\mathbf{c u r l}, \Omega_{\mathrm{C}}\right)$ and $\mathbf{g r a d} \widetilde{\Phi} \in \mathrm{H}^{r}\left(\Omega_{\mathrm{D}}\right)^{3}$, with $r \in\left(\frac{1}{2}, 1\right]$.

If $\left(\left(\mathbf{H}_{h}, \widetilde{\Phi}_{h}, I_{h}\right), \lambda_{h}\right)$ is solution of problem $\mathbf{D L P}$, then $\left(\mathbf{H}_{h}, \widetilde{\Phi}_{h}\right)$ is solution of problem DHP.

Conversely, if $\left(\mathbf{H}_{h}, \widetilde{\Phi}_{h}\right)$ is solution of problem DHP and $I_{h j}=\llbracket \widetilde{\Phi}_{h} \rrbracket_{\Sigma_{j}}, j=$ $1, \ldots, J$, then, there exists $\lambda_{h} \in \mathcal{L}_{h}\left(\Gamma_{\mathrm{D}}\right) / \mathbb{C}$ such that $\left(\left(\mathbf{H}_{h}, \widetilde{\Phi}_{h}, I_{h}\right), \lambda_{h}\right)$ is solution of problem DLP.

Proof. Let $\left(\left(\mathbf{H}_{h}, \widetilde{\Phi}_{h}, I_{h}\right), \lambda_{h}\right)$ be solution of problem DLP. Since $\left(\mathbf{H}_{h}, \widetilde{\Phi}_{h}, I_{h}\right) \in$ $\mathcal{Z}_{h}$, then $\left(\mathbf{H}_{h}, \widetilde{\Phi}_{h}\right) \in \mathcal{W}_{h}$ (because of Lemma 6.1) and $I_{h j}=\llbracket \widetilde{\Phi}_{h} \rrbracket_{\Sigma_{j}}, j=1, \ldots, J$. Therefore, because of (6.7), $\widetilde{\Phi}_{h}$ satisfies assumption (6.4) in Lemma 6.2. Then, (6.9) implies (5.8).

On the other hand, let $\left(\mathbf{G}_{h}, \widetilde{\Psi}_{h}\right) \in \mathcal{W}_{h}^{0}$ and $c_{h j}=\llbracket \widetilde{\Psi}_{h} \rrbracket_{\Sigma_{j}}, j=1, \ldots, J$. Because of Lemma 6.1 and equation $(6.2),\left(\mathbf{G}_{h}, \widetilde{\Psi}_{h}, c_{h}\right) \in \mathcal{Z}_{h}^{0}$. Since $\operatorname{grad} \widetilde{\Psi}_{h} \times \mathbf{n}=\mathbf{0}$ on $\Gamma_{\mathrm{D}}$, then, because of (6.3), we have $\operatorname{grad}_{\Gamma}\left(\left.\widetilde{\Psi}_{h}\right|_{\Gamma_{\mathrm{D}}}\right)=\mathbf{0}$. Therefore, by testing (6.8) with such $\left(\mathbf{G}_{h}, \widetilde{\Psi}_{h}, c_{h}\right)$, we obtain (5.9). Thus, $\left(\mathbf{H}_{h}, \widetilde{\Phi}_{h}\right)$ is solution of problem DHP.

Conversely, let $\left(\mathbf{H}_{h}, \widetilde{\Phi}_{h}\right)$ be solution of problem DHP. Since the assumptions of Corollary 5.7 are fulfilled, this solution is unique. On the other hand, Theorem 6.3 shows that there exists also a unique solution $\left(\left(\mathbf{H}_{h}^{\prime}, \widetilde{\Phi}_{h}^{\prime}, I_{h}\right), \lambda_{h}\right)$ of problem DLP. But, then, we have already proved that $\left(\mathbf{H}_{h}^{\prime}, \widetilde{\Phi}_{h}^{\prime}\right)$ is solution of problem DHP. Hence, $\left(\mathbf{H}_{h}^{\prime}, \widetilde{\Phi}_{h}^{\prime}\right)=\left(\mathbf{H}_{h}, \widetilde{\Phi}_{h}\right)$, and we conclude the proof.

Problem DLP is the one we have actually implemented. The degrees of freedom for this problem are the following ones: 
- for $\mathbf{H}_{h} \in \mathcal{N}_{h}\left(\Omega_{\mathrm{C}}\right): \int_{\ell} \mathbf{H}_{h} \cdot \mathbf{t}_{\ell} d \gamma, \forall$ edge $\ell \subset \bar{\Omega}_{\mathrm{C}} \backslash \Gamma_{\mathrm{I}}$;

- for $\widetilde{\Phi}_{h} \in \mathcal{L}_{h}\left(\widetilde{\Omega}_{\mathrm{D}}\right) / \mathbb{C}: \widetilde{\Phi}_{h}(P), \forall$ vertex $P \in \bar{\Omega}_{\mathrm{D}}$ (one of them fixed to zero).

- for $I_{h} \in \mathbb{C}^{J}: I_{h j}, j=1, \ldots, J\left(I_{h K+1}, \ldots, I_{h J}\right.$ are directly computed from $\left.\mathbf{f}\right)$;

- for $\lambda_{h} \in \mathcal{L}_{h}\left(\Gamma_{\mathrm{D}}\right) / \mathbb{C}: \lambda_{h}(P), \forall$ vertex $P \in \Gamma_{\mathrm{D}}$ (one of them fixed to zero).

Remark 6.2. We have imposed the boundary condition of problem DHP by means of a Lagrange multiplier. However, this is not the only way of doing it. An alternative procedure consists of using the fact that, for each edge $\ell \subset \Gamma_{\mathrm{D}}$,

$$
\begin{aligned}
\widetilde{\Phi}_{h}\left(P_{\ell}^{+}\right)-\widetilde{\Phi}_{h}\left(P_{\ell}^{-}\right) & =\int_{\ell} \operatorname{grad} \widetilde{\Phi}_{h} \cdot \mathbf{t}_{\ell} d \gamma=\int_{\ell} \mathbf{n} \times\left(\operatorname{grad} \widetilde{\Phi}_{h} \times \mathbf{n}\right) \cdot \mathbf{t}_{\ell} d \gamma \\
& =\int_{\ell} \mathbf{n} \times \mathbf{f}_{\mathrm{I}} \cdot \mathbf{t}_{\ell} d \gamma=\int_{\ell} \mathbf{n} \times \mathbf{f} \cdot \mathbf{t}_{\ell} d \gamma
\end{aligned}
$$

Therefore, the values of $\widetilde{\Phi}_{h}(P)$ can be obtained for each vertex $P \in \Gamma_{\mathrm{D}}$, by the following procedure:

1. fix arbitrarily the value of $\widetilde{\Phi}_{h}$ at a given vertex $P_{0} \in \Gamma_{\mathrm{D}}$ :

$$
\widetilde{\Phi}_{h}\left(P_{0}\right)=0
$$

(this can be done because $\left.\widetilde{\Phi}_{h} \in \Theta_{h} / \mathbb{C}\right)$;

2. for each other vertex $P \in \Gamma_{\mathrm{D}}$ (those on $\Gamma_{\mathrm{D}} \cap \Sigma_{j}, j=K+1, \ldots, J$, must be counted twice):

(a) find a path $\Gamma_{P}$ joining $P_{0}$ with $P$, which does not cross any $\Sigma_{j} \cap \Gamma_{\mathrm{D}}$, $j=K+1, \ldots, J$, and which consists of adequately oriented edges $\ell \subset \Gamma_{\mathrm{D}}$ :

$$
\Gamma_{P}:= \pm \ell_{1} \cup \cdots \cup \pm \ell_{N_{P}}
$$

(b) evaluate:

$$
\widetilde{\Phi}_{h}(P)= \pm \int_{\ell_{1}} \mathbf{n} \times \mathbf{f} \cdot \mathbf{t}_{\ell_{1}} d \gamma \pm \ldots \pm \int_{\ell_{N_{P}}} \mathbf{n} \times \mathbf{f} \cdot \mathbf{t}_{\ell_{N_{P}}} .
$$

The main drawback of this procedure is that step 2(a) is rather complicate to implement (see [11]). The strategy we have proposed is more expensive than this one in terms of degrees of freedom (one unknown per vertex on $\Gamma_{\mathrm{D}}$ is added, instead of being eliminated). Nevertheless, one neat advantage is that its implementation is quite straightforward.

7. Numerical experiments. In this section we present some numerical results obtained with a code developed by us, which implements in MAtLaB the method described above.

We have solved a particular problem with known analytical solution to validate the computer code and to test the performance and convergence properties of the method. The geometry of the domain is similar to that of an electric furnace with only one electrode.

More precisely, we consider a domain $\Omega$ containing a conductor $\Omega_{\mathrm{C}}$ and dielectric $\Omega_{\mathrm{D}}$ as shown in Figure 7.1.

We assume that $\bar{\Omega}_{\mathrm{C}}$ and $\bar{\Omega}=\bar{\Omega}_{\mathrm{C}} \cup \bar{\Omega}_{\mathrm{D}}$ are coaxial cylinders of radius $R_{\mathrm{C}}$ and $R_{\mathrm{D}}$, respectively, and height $L$. To obtain the data for a test problem in this domain with known analytical solution, we consider that $\Omega_{\mathrm{C}}$ and $\Omega$ are bounded sections of respective infinite cylinders. The electric conductivity $\sigma$ is taken as constant in $\Omega_{\mathrm{C}}$ 


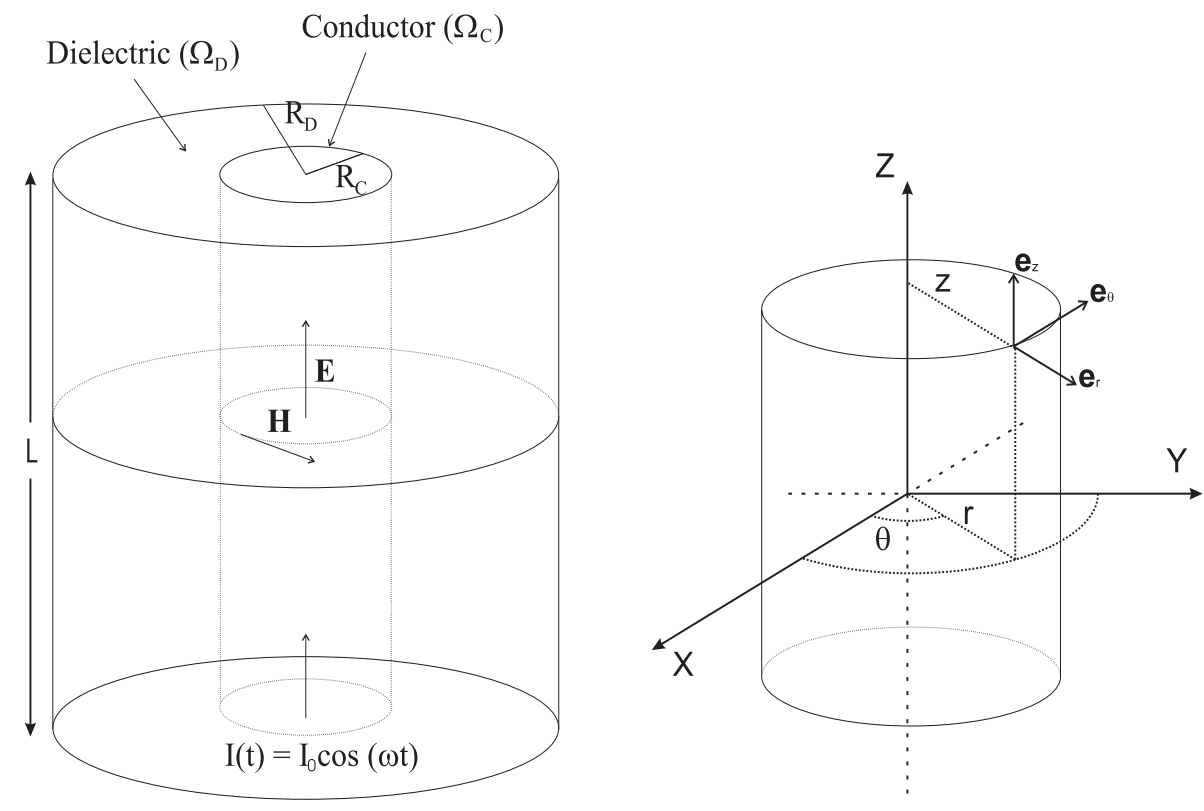

FIG. 7.1. Sketch of the domain. Coordinate system.

and the magnetic permeability $\mu$, constant in the whole $\Omega$. We consider that an alternating current $\mathbf{J}$ goes through the conductor $\Omega_{\mathrm{C}}$ in the direction of its axis; this current is assumed to be axially symmetric with an intensity $I(t)=I_{0} \cos (\omega t)$.

We analyze this problem using a cylindrical coordinate system $(r, \theta, z)$ with the $z$-axis coinciding with the common axis of both cylinders (see Figure 7.1). We denote $\mathbf{e}_{r}, \mathbf{e}_{\theta}$, and $\mathbf{e}_{z}$ the unit vectors in the corresponding coordinate directions.

Because of the assumed conditions on $\mathbf{J}$, only the $z$-component of the electric field $\mathbf{E}=\frac{1}{\sigma} \mathbf{J}$ does not vanish in the conductor. Moreover, it depends on the radial coordinate $r$, but is independent of the other two coordinates $z$ and $\theta$. Consequently, only the $\theta$-component of the magnetic field $\mathbf{H}=\frac{\mathrm{i}}{\omega \mu} \mathbf{c u r l} \mathbf{E}$ does not vanish and it also depends only on the coordinate $r$. Then, taking into account the expression of the curl operator in cylindrical coordinates, we have $\mathbf{H}(r, \theta, z)=H_{\theta}(r) \mathbf{e}_{\theta}$, with $H_{\theta}$ satisfying the equation

$$
\mathrm{i} \omega \mu H_{\theta}(r)-\frac{d}{d r}\left\{\frac{1}{\sigma r} \frac{d}{d r}\left[r H_{\theta}(r)\right]\right\}=0, \quad r \in\left(0, R_{\mathrm{C}}\right),
$$

and the boundary conditions

$$
\left|H_{\theta}(0)\right|<\infty, \quad H_{\theta}\left(R_{\mathrm{C}}\right)=\frac{I_{0}}{2 \pi R_{\mathrm{C}}} .
$$

To solve this problem, we perform the change of variable $x=\gamma r$, where $\gamma=$ $\sqrt{i \omega \mu \sigma} \in \mathbb{C}$. Then, we obtain the equation

$$
x^{2} \frac{d^{2}}{d x^{2}} \widetilde{H}_{\theta}(x)+x \frac{d}{d x} \widetilde{H}_{\theta}(x)-\left(x^{2}+1\right) \widetilde{H}_{\theta}(x)=0, \quad x \in\left(0, \gamma R_{\mathrm{C}}\right),
$$

where $\widetilde{H}_{\theta}(x)=H_{\theta}(x / \gamma)$. 
This is a Bessel equation, the solution of which is given by $\widetilde{H}_{\theta}(x)=\alpha \mathrm{I}_{1}(x)$, with $\mathrm{I}_{1}$ being the modified Bessel function of the first kind and $\alpha$ a constant to be obtained from the boundary condition at $x=\gamma R_{\mathrm{C}}$. Thus, the magnetic field in the conductor is given by

$$
\mathbf{H}(r, \theta, z)=\frac{I_{0}}{2 \pi R_{\mathrm{C}}} \frac{\mathrm{I}_{1}(\gamma r)}{\mathrm{I}_{1}\left(\gamma R_{\mathrm{C}}\right)} \mathbf{e}_{\theta}, \quad r \in\left(0, R_{\mathrm{C}}\right), \quad \theta \in[0,2 \pi], \quad z \in \mathbb{R} .
$$

On the other hand, the magnetic field created by an infinite circular cylindrical conductor of radius $R_{\mathrm{C}}$ carrying an axially aligned and symmetric current of intensity $I_{0}$, is computed using the Ampère's circuital law (see for instance [28]). In cylindrical coordinates it is given by $\mathbf{H}(r, \theta, z)=H_{\theta}(r) \mathbf{e}_{\theta}$, with

$$
H_{\theta}(r)=\frac{I_{0}}{2 \pi r}, \quad r \geq R_{\mathrm{C}} .
$$

Once more, the magnitude of $H_{\theta}$ depends only on the radial coordinate $r$.

Moreover, from this expression, it is also possible to know the multivalued magnetic potential $\widetilde{\Phi}$ which corresponds to the magnetic field in the dielectric domain. Indeed, taking into account the expression of the gradient operator in cylindrical coordinates, we obtain

$$
\widetilde{\Phi}(r, \theta, z)=\frac{I_{0}}{2 \pi} \theta, \quad r>R_{\mathrm{C}}, \quad \theta \in[0,2 \pi], \quad z \in \mathbb{R} .
$$

Notice that the scalar potential depends only on the variable $\theta$ and experiments a jump of magnitude $I_{0}$ across the cut surface $\Sigma$ placed at $\theta=0$.

Now, we consider again the bounded cylinder of Figure 7.1. The boundary conditions added to define properly this problem are the following:

- on the exterior boundary of the dielectric domain (i.e., the lateral surface of the cylinder $\Omega$ and the outer part of its top and bottom surfaces) we consider the condition $\mathbf{H} \times \mathbf{n}=\mathbf{f}$, with $\mathbf{f}$ being obtained from the analytical solution:

$$
\begin{aligned}
& \mathbf{f}\left(R_{\mathrm{D}}, \theta, z\right)=-\frac{I_{0}}{2 \pi R_{\mathrm{D}}} \mathbf{e}_{z}, \quad \theta \in[0,2 \pi], \quad z \in\left[-\frac{L}{2}, \frac{L}{2}\right], \\
& \mathbf{f}\left(r, \theta, \pm \frac{L}{2}\right)= \pm \frac{I_{0}}{2 \pi R_{\mathrm{C}}} \frac{\mathrm{I}_{1}(\gamma r)}{\mathrm{I}_{1}\left(\gamma R_{\mathrm{C}}\right)} \mathbf{e}_{r}, \quad r \in\left[R_{\mathrm{C}}, R_{\mathrm{D}}\right], \quad \theta \in[0,2 \pi] ;
\end{aligned}
$$

- on the top and bottom surfaces of the conducting cylinder $\Omega_{\mathrm{C}}$, we impose

$$
\mathbf{E}\left(r, \theta, \pm \frac{L}{2}\right) \times \mathbf{n}=\mathbf{0}, \quad r \in\left(0, R_{\mathrm{D}}\right), \quad \theta \in[0,2 \pi] ;
$$

which is true in this case, because the electric field has vanishing $r$ - and $\theta$-components and, thus, it aligns with the normal vector $\mathbf{n}$ on these surfaces.

Finally, we have used the following geometrical and physical data:

- $R_{\mathrm{C}}=1 \mathrm{~m} ;$

- $R_{\mathrm{D}}=2 \mathrm{~m}$

- $L=1 \mathrm{~m}$

- $\sigma=151565.8(\Omega \mathrm{m})^{-1}$;

- $\mu=\mu_{0}=4 \pi 10^{-7} \mathrm{Hm}^{-1}$ (magnetic permeability of free space);

- $I_{0}=62000 \mathrm{~A}$;

- $\omega=50 \mathrm{~Hz}$. 
To determine the order of convergence, the numerical method has been used on several successively refined meshes and we have compared the obtained numerical solutions with the analytical one. Figures 7.2 and 7.3 show the coarsest meshes used for conductor and dielectric domains, respectively.

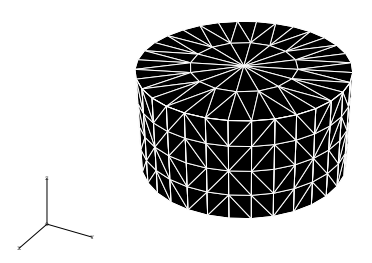

FIG. 7.2. Coarsest mesh on the conductor domain.

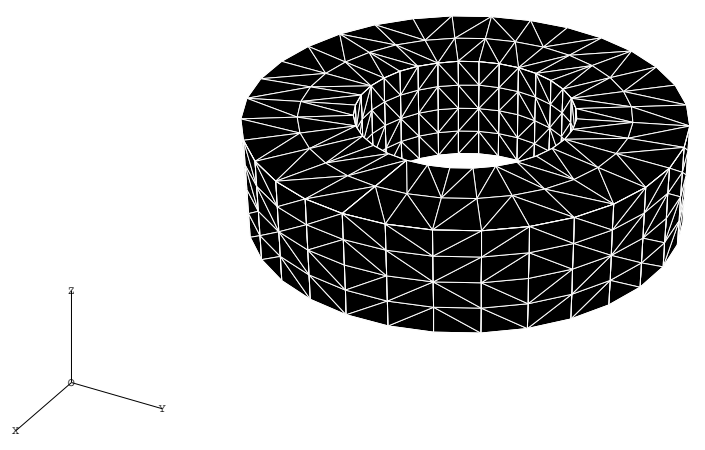

FIG. 7.3. Coarsest mesh on the dielectric domain.

Table 7.1 shows the $\mathrm{H}(\mathbf{c u r l}, \Omega)$-norms of the approximate solutions $\mathbf{H}_{h}$ computed on several meshes and their corresponding errors. The total number of degrees of freedom (d.o.f.) for each mesh are also included.

TABLE 7.1

$\mathrm{H}(\mathbf{c u r l}, \Omega)$-norm of errors and exact solution.

\begin{tabular}{|c|c|c|c|}
\hline Mesh-size & Number d.o.f. & Computed solution & Error \\
\hline$h$ & 1699 & 62257.66 & 36504.31 \\
$h / 2$ & 11285 & 64063.20 & 23591.63 \\
$h / 3$ & 35671 & 64296.90 & 16646.00 \\
$h / 4$ & 81769 & 64363.90 & 12709.90 \\
$h / 5$ & 156491 & 64391.83 & 10228.25 \\
\hline
\end{tabular}

Figure 7.4 shows a log-log plot of the errors measured in $\mathrm{H}(\mathbf{c u r l}, \Omega)$-norm versus the number of d.o.f. for the same meshes. A linear dependence on the mesh-size is obtained by calculating the slope of the line. These $\mathcal{O}(h)$ errors agree with the 
theoretical results, since the solution is smooth and, hence, the hypotheses of Theorem 5.4 are fulfilled for $r=1$.

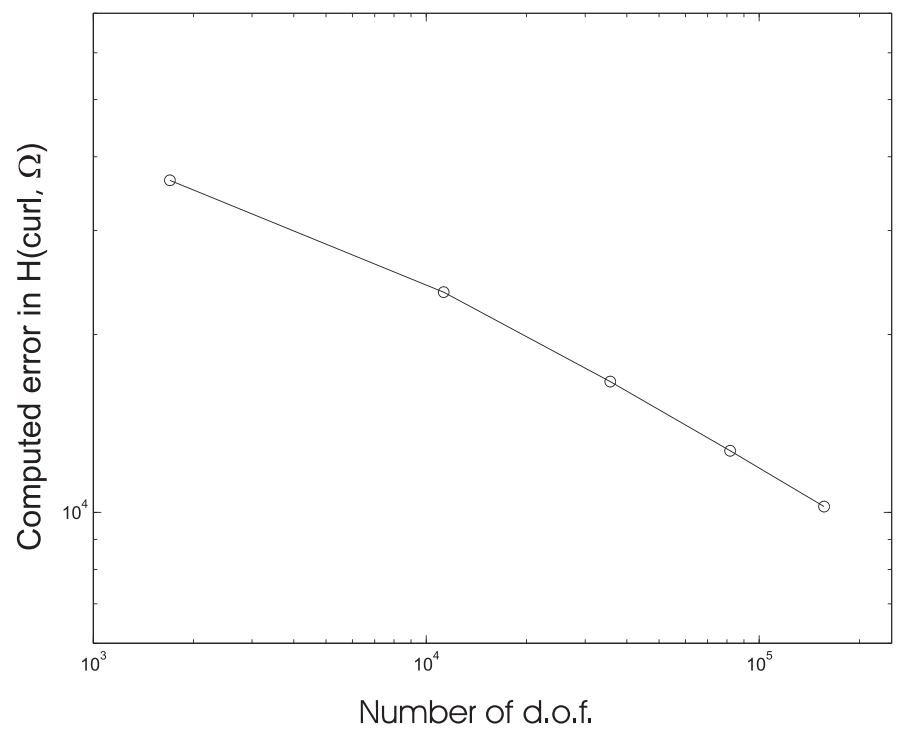

FIG. 7.4. Error versus number of d.o.f. (log-log scale).

Finally, Figures 7.5 and 7.6 show the intensity of the computed magnetic field $\left|\mathbf{H}_{h}\right|$ in the conductor domain $\Omega_{\mathrm{C}}$ and the computed magnetic potential $\widetilde{\Phi}_{h}$ in the dielectric domain $\Omega_{\mathrm{D}}$. The former is presented in a section of $\Omega_{\mathrm{C}}$ to show its behavior in the interior of this domain.

Acknowledgments. The authors thank Professor Alain Bossavit for many valuable discussions.

\section{REFERENCES}

[1] A. Alonso And A. VAlli, Some remarks on the characterization of the space of tangential traces of $\mathrm{H}(\mathbf{c u r l}, \Omega)$ and the construction of an extension operator, Manuscripta Math., 89 (1996), pp. 159-178.

[2] — A domain decomposition approach for heterogeneous time-harmonic Maxwell equations, Comput. Methods Appl. Mech. Engrg., 143 (1997), pp. 97-112.

[3] — An optimal domain decomposition preconditioner for low-frequency time-harmonic Maxwell equations, Math. Comp., 68 (1999), pp. 607-631.

[4] H. Ammari, A. Buffa, and J. C. NÉdÉlec, A justification of eddy currents model for the Maxwell equations, SIAM J. Appl. Math., 60 (2000), pp. 1805-1823.

[5] C. Amrouche, C. Bernardi, M. Dauge, and V. Girault, Vector potentials in threedimensional non-smooth domains, Math. Meth. Appl. Sci, 21 (1998), pp. 823-864.

[6] A. Bermúdez, J. Bullón, and F. Pena, A finite element method for the thermoelectrical modelling of electrodes, Comm. Numer. Methods Engrg., 14 (1998), pp. 581-593.

[7] A. Bermúdez, M. C. Muñiz, F. Pena, And J. Bullón, Numerical computation of the electromagnetic field in the electrodes of a three-phase arc furnace, Internat. J. Numer. Methods Engrg., 46 (1999), pp. 649-658.

[8] A. Bossavit, Magnetostatic problems in multiply connected regions: some properties of the curl operator, IEE Proc., 135, Pt. A (1988), pp. 179-187.

[9] — Whitney forms: a class of finite elements for three-dimensional computations in electromagnetism, IEE Proc., 135, Pt. A (1988), pp. 493-500. 


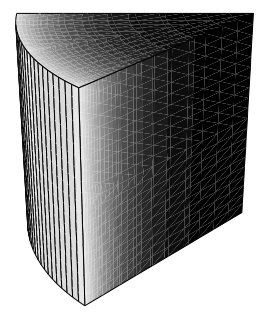

FIG. 7.5. Intensity of the magnetic field $\left|\mathbf{H}_{h}\right|$ in the conductor.

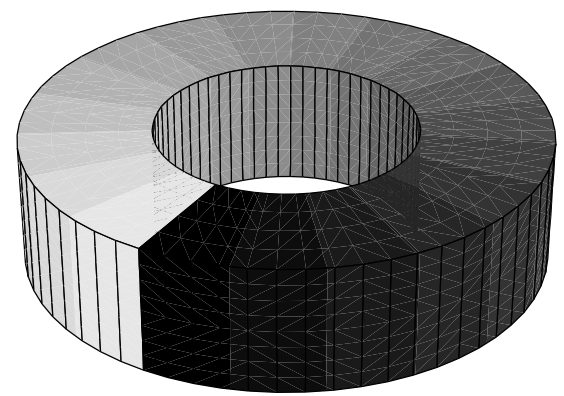

FIG. 7.6. Magnetic potential $\widetilde{\Phi}_{h}$ in the dielectric.

[10] - The computation of eddy-currents in dimension 3 by using mixed finite elements and boundary elements in association, Math. Comput. Modelling, 15 (1991), pp. 33-42.

[11] - Computational Electromagnetism. Variational Formulations, Complementarity, Edge Elements. Academic Press. San Diego, CA, 1998.

[12] — "Hybrid" electric-magnetic methods in eddy-current problems, Comput. Methods Appl. Mech. Engrg., 178 (1999), pp. 383-39.

[13] A. Bossavit and J. C. VÉrité, A mixed FEM-BIEM method to solve 3-D eddy current problems, IEEE Trans. Magn., 18 (1982), pp. 431-435.

[14] A. Buffa And P. Ciarlet JR., On traces for functional spaces related to Maxwell's equations. I. An integration by parts formula in Lipschitz polyhedra. Math. Methods Appl. Sci., 24 (2001), pp. 9-30.

[15] - On traces for functional spaces related to Maxwell equations. II. Hodge decompositions on the boundary of Lipschitz polyhedra and applications, Math. Methods Appl. Sci., 24 (2001), pp. 31-48.

[16] M. Costabel, V. J. Ervin, and E. P. Stephan, Symmetric coupling of finite elements and boundary elements for a parabolic-elliptic interface problem, Quart. Appl. Math., 48 (1990), pp. 265-279.

[17] P. Fernandes and G. Gilard, Magnetostatic and electrostatic problems in inhomogeneous anisotropic media with irregular boundary and mixed boundary conditions, Math. Models Methods Appl. Sci., 7 (1997), pp. 957-991.

[18] S. I. HaRiharan AND R. C. MACCAMY, An integral equation procedure for eddy current prob- 
lems, J. Comput. Phys., 45 (1982), pp. 80-99.

[19] V. Girault And P. A. Raviart, Finite Element Methods for Navier-Stokes Equations. Theory and Algorithms. Springer-Verlag, Berlin, 1986.

[20] L. Kettunen, K. Forsman, And A. Bossavit, Formulation of the eddy current problem in multiple connected regions in terms of $h$, Internat. J. Numer. Methods Engrg., 41 (1998), pp. 935-954.

[21] R. C. MacCamy and E. Stephan, A skin effect approximation for eddy current problems, Arch. Rational Mech. Anal., 90 (1985), pp. 87-98.

[22] R. C. MacCamy and M. Suri, A time-dependent interface problem for two-dimensional eddy currents, Quart. Appl. Math., 44 (1987), pp. 675-690.

[23] P. Monk, A mixed method for approximating Maxwell's equations, SIAM J. Numer. Anal., 28 (1991), pp. 1610-1634.

[24] — A finite element method for approximating the time-harmonic Maxwell equations, Numer. Math., 63 (1992), pp. 243-261.

[25] — - Analysis of a finite element method for Maxwell's equations, SIAM J. Numer. Anal., 29 (1992), pp. 714-729.

[26] J. C. NÉDÉLEC, Mixed finite elements in $\mathbb{R}^{3}$, Numer. Math., 35 (1980), pp. 315-341.

[27] R. PicARD, On the boundary value problems of electro- and magnetostatics, Proc. Roy. Soc. Edimb., Sect. A, 92 (1982), pp. 165-174.

[28] B. D. Popović, Introductory Engineering Electromagnetics, Addison Wesley, 1971.

[29] P. P. Silvester and R. L. Ferrari, Finite Elements for Electrical Engineers, Cambridge University Press, Cambridge, 1996.

[30] I. A. Tsukerman, Error estimation for finite element solutions of the eddy currents problem, COMPEL, 9 (1990), pp. 83-98. 